\title{
Fourier type transforms on Lie symmetry groups
}

\author{
Mark Craddock ${ }^{a}$ \\ School of Mathematical and Physical Sciences, University of Technology Sydney, \\ P.O. Box 123, Broadway, New South Wales 2007, Australia
}

(Received 24 July 2014; accepted 16 August 2015; published online 1 September 2015)

\begin{abstract}
In this paper, we construct operators on a Lie symmetry group which may be regarded as Fourier transforms. Essentially, we integrate solutions generated by Lie symmetries against suitable test functions. We show that this idea leads to a powerful method for solving Cauchy problems for parabolic and hyperbolic equations in two and higher dimensions. We also discuss applications to the elliptic case. (C) 2015 AIP Publishing LLC. [http://dx.doi.org/10.1063/1.4929653]
\end{abstract}

\section{INTRODUCTION}

Lie symmetry methods provide a powerful set of tools for the solution of partial differential equations (PDEs). For initial value problems, the standard technique has long been to seek a symmetry which preserves the initial data, then use the fact that the solution of the PDE satisfying the desired conditions will be invariant under the action of the symmetry. See, for example, Lie, ${ }^{21}$ Olver, ${ }^{23}$ and Bluman and Kumei. ${ }^{4}$

However, other methods are also available. One approach to the solution of initial value problems for parabolic PDEs is to seek an integral transform of a fundamental solution obtained by applying a symmetry to a stationary solution. This provides a link between harmonic analysis and Lie symmetries. This is a topic that has been studied in recent years, in works such as Refs. 5, 6, 11, $9,10,25$, and 15 . In this paper, we are concerned with extending this link.

The basic question we address is the following: suppose that $\tilde{u}(x ; \epsilon), \epsilon \in \mathbb{R}$, is a family of solutions of a linear PDE obtained by symmetry from a known solution $u$. How should we use $\tilde{u}(x ; \epsilon)$ ? Our answer is that $\tilde{u}(x ; \epsilon)$ should be regarded as the kernel of an integral transform. To see why, observe that if $\Omega$ is an appropriate region of integration and $\varphi$ is a suitable test function, then

$$
U(x)=\int_{\Omega} \varphi(\epsilon) \tilde{u}(x ; \epsilon) d \epsilon
$$

will again be a solution.

In other words, in the linear case, the family of solutions $\tilde{u}(x ; \epsilon)$ provides the kernel of an integral operator which maps elements of an appropriate function space to solutions of the PDE. In essence, we can think of (1.1) as a type of Fourier transform over a one parameter symmetry group.

If the symmetry is sufficiently complex, then new solution (1.1) allows us to construct explicit solutions to certain problems, without the need to solve another differential equation, as say, the method of group invariant solutions does. In Refs. 12, 11, and 8, this idea was used to construct integral transforms of fundamental solutions of parabolic PDEs from a stationary solution. In this paper, we extend the method to include Fourier analysis with scaling symmetries and apply the general theory to hyperbolic and elliptic equations.

While we must emphasize that we do not yet have a complete theory, the methods outlined by our examples should be applicable to a wide variety of problems. There remain many open questions in this area. The paper notes some of these, but see, for instance, the comment at the end of Example 3.5. It is also worth mentioning that the application of the method to elliptic boundary

\footnotetext{
a)E-mail: Mark.Craddock@uts.edu.au
} 
value problems requires development. We hope that the current work will stimulate further research in this area.

\section{FOURIER TRANSFORMS ON GROUPS}

We briefly sketch the background material. Suppose that $G$ is a locally compact group. Then, it is well known that it possesses a left invariant Haar measure $\mu$. So that one may define $L^{1}(G)$ in the natural way and the Haar integral $\int_{G} f(g) d \mu(g), f \in L^{1}(G)$ may be constructed analogously to the Lebesgue integral. See Ref. 24 for Haar measure and integrals.

Next, consider a unitary representation $\rho$ of $G$, defined on some Hilbert space $\mathcal{H}$. For $f \in$ $L^{1}(G)$, we may form the Fourier transform

$$
\rho(f)=\int_{G} f(g) \rho(g) d \mu(g) .
$$

This defines an operator on the Hilbert space $\mathcal{H}$ by

$$
\rho(f) v=\int_{G} f(g) \rho(g) v d \mu(g), v \in \mathcal{H} .
$$

The group Fourier transform has been extensively studied. The literature is very substantial and it is not our intention to present a detailed discussion of the subject, but see Refs. 24, 17, and 28 for an introduction.

For linear PDEs, it is often possible to realise the symmetries as global representations of the underlying Lie group in the following sense. One has a Lie group $\mathcal{G}$, a representation $(\sigma, V)$ of $\mathcal{G}$, and a mapping $A: V \rightarrow H$, where $H$ is a solution space of the equation. Then, if the action of the symmetries on solutions $u=A f$ is denoted by $\rho(g) u$ for $g \in \mathcal{G}$, the following relationship holds:

$$
(\rho(g) A f)(x)=(A \sigma(g) f)(x), g \in \mathcal{G},
$$

where $f \in V$. (See Refs. 6, 7, and 9 for more on this.) As a consequence, if we take $\mathbf{v}_{k}$ to be a vector field in the Lie symmetry algebra of the PDE, then the operator

$$
v_{k}(x)=\int_{A} \varphi(\epsilon) \rho\left(\exp \left(\epsilon \mathbf{v}_{k}\right)\right) u(x) d \epsilon
$$

is essentially a group theoretic Fourier transforms, which inherits properties of the representations equivalent to $\rho$. We note that the representation $\sigma$ will often not be unitary, though this will not concern us here. Operator (2.3) is precisely the operator appearing in (1.1). It is therefore reasonable to expect that the Fourier transform on the symmetry group of a PDE will provide us with a useful tool for studying solutions of the PDE.

Let us briefly recall how the method works in the parabolic case. We illustrate with the one-dimensional heat equation. Let $\mathbf{v}_{k}, k=1, \ldots, 6$ be the basis for the Lie algebra of symmetries of the heat equation given in Chapter 2 of Ref. 23. From the solution $\left(\prod_{k=1}^{6} \rho\left(\exp \left(\epsilon \mathbf{v}_{k}\right) u_{0}\right)\right)(x, t)$, we obtain the Fourier transform

$$
\begin{aligned}
& \left(\rho(\varphi) u_{0}\right)(x, t)=\int_{\mathbb{R}^{6}} \frac{\varphi\left(\epsilon_{1}, \epsilon_{2}, \epsilon_{3}, \epsilon_{4}, \epsilon_{5}, \epsilon_{6}\right)}{\sqrt{1+4 \epsilon_{6} t}} \exp \left(\epsilon_{3}-\frac{\epsilon_{5} x+\epsilon_{6} x^{2}-\epsilon_{5}^{2} t}{1+4 \epsilon_{6} t}\right) \\
& \times u_{0}\left(\frac{e^{-\epsilon_{4}}\left(x-2 \epsilon_{5} t\right)}{1+4 \epsilon_{6} t}-\epsilon_{1}, \frac{e^{-2 \epsilon_{4} t}}{1+4 \epsilon_{6} t}-\epsilon_{2}\right) d \epsilon_{1} d \epsilon_{2} d \epsilon_{3} d \epsilon_{4} d \epsilon_{5} d \epsilon_{6} .
\end{aligned}
$$

This is an operator which maps solutions $u_{0}$ to solutions $\left(\rho(\varphi) u_{0}\right)(x, t)$. We will not attempt to analyze this operator in full detail. Rather we will consider a special case. We take $\epsilon_{k}=0$, $k=1, \ldots, 5$, set $\epsilon_{6}=\epsilon$, and thus study a Fourier transform over a one parameter symmetry group.

We use this group Fourier transform to solve

$$
u_{t}=u_{x x}, x \geq 0, u(x, 0)=f(x), u(0, t)=0 .
$$

We will take $u(x, t)=x$ and define $\left(\rho(\varphi) u_{0}\right)(x, t)=u(x, t)$ and the group Fourier transform becomes

$$
u(x, t)=\int_{0}^{\infty} \frac{x \varphi(\epsilon)}{(1+4 \epsilon t)^{3 / 2}} \exp \left(-\frac{\epsilon x^{2}}{1+4 \epsilon t}\right) d \epsilon
$$


for suitable $\varphi$. In order for this to make sense, we require $\varphi$ to decay fast enough to guarantee that the integral is convergent and that we can differentiate under the integral sign. Clearly, $u(0, t)=0$ and $u(0, t)=x \Phi\left(x^{2}\right)$, where $\Phi$ is the Laplace transform of $\varphi$.

We will use the Laplace transform

$$
\begin{aligned}
\frac{x}{(1+4 \epsilon t)^{3 / 2}} \exp \left(-\frac{\epsilon x^{2}}{1+4 \epsilon t}\right) & =\frac{1}{\sqrt{4 \pi t}} \int_{0}^{\infty} \exp \left(-\frac{x^{2}+\xi}{4 t}-\epsilon \xi\right) \\
& \times \sinh \left(\frac{\sqrt{\xi} x}{2 t}\right) d \xi
\end{aligned}
$$

which may be easily verified using standard tables, such as those in Ref. 1.

So after reversing the order of integration (2.6) is

$$
\begin{aligned}
u(x, t) & =\frac{1}{\sqrt{4 \pi t}} \int_{0}^{\infty} \int_{0}^{\infty} \varphi(\epsilon) \exp \left(-\frac{x^{2}+\xi}{4 t}-\epsilon \xi\right) \sinh \left(\frac{\sqrt{\xi} x}{2 t}\right) d \epsilon d \xi \\
& =\frac{1}{\sqrt{4 \pi t}} \int_{0}^{\infty} \Phi(\xi) \exp \left(-\frac{x^{2}+\xi}{4 t}\right) \sinh \left(\frac{\sqrt{\xi} x}{2 t}\right) d \xi \\
& =\frac{1}{\sqrt{\pi t}} \int_{0}^{\infty} y \Phi\left(y^{2}\right) \exp \left(-\frac{x^{2}+y^{2}}{4 t}\right) \sinh \left(\frac{y x}{2 t}\right) d y
\end{aligned}
$$

and $u(x, 0)=f(x)=x \Phi\left(x^{2}\right)$. This is the solution usually obtained by the method of images

If we were to take $u_{0}=1, \epsilon_{5}=i \epsilon$, and $\epsilon_{k}=0$ otherwise, we have the integral kernel operator

$$
\left(\rho(\varphi) u_{0}\right)(x, t)=\int_{-\infty}^{\infty} \varphi(\epsilon) e^{-i \epsilon x-\epsilon^{2} t} d \epsilon,
$$

which is the form of solution usually obtained by the classical Fourier transform. Thus, the classical Fourier analysis of the heat equation is essentially contained in our symmetry group Fourier transform.

Fundamental solutions of linear parabolic equations on the line can be obtained by this method whenever the symmetry group is at least four dimensional. ${ }^{9}$ The extension to higher dimensional parabolic problems was given in Ref. 10. We can also solve linear systems using the same approach.

Example 2.1. We wish to solve the linear system

$$
u_{t}=u_{x x}-\frac{A^{2}}{x^{2}} v, A \neq 0, \quad v_{t}=v_{x x}-\frac{B^{2}}{x^{2}} u, B \neq 0,
$$

subject to $u(x, 0)=f(x), v(x, 0)=g(x)$. It is not hard to show that this system possesses the symmetry

$$
\begin{aligned}
& \tilde{u}_{\epsilon}(x, t)=\frac{1}{\sqrt{1+4 \epsilon t}} \exp \left(-\frac{\epsilon x^{2}}{1+4 \epsilon t}\right) u\left(\frac{x}{1+4 \epsilon t}, \frac{t}{1+4 \epsilon t}\right), \\
& \tilde{v}_{\epsilon}(x, t)=\frac{1}{\sqrt{1+4 \epsilon t}} \exp \left(-\frac{\epsilon x^{2}}{1+4 \epsilon t}\right) v\left(\frac{x}{1+4 \epsilon t}, \frac{t}{1+4 \epsilon t}\right) .
\end{aligned}
$$

If $\mu=\frac{1}{2} \sqrt{1+4 A B}$, then a stationary solution of the system is $u(x, t)=x^{\frac{1}{2}+\mu}$ and $v(x, t)=B / A x^{\frac{1}{2}+\mu}$. Thus,

$$
\begin{aligned}
U(x, t, \epsilon) & =\frac{x^{1 / 2+\mu}}{(1+4 \epsilon t)^{1+\mu}} \exp \left(-\frac{\epsilon x^{2}}{1+4 \epsilon t}\right), \\
V(x, t, \epsilon) & =\frac{B}{A} \frac{x^{1 / 2+\mu}}{(1+4 \epsilon t)^{1+\mu}} \exp \left(-\frac{\epsilon x^{2}}{1+4 \epsilon t}\right)
\end{aligned}
$$

are a solution. We may then form a solution by setting

$$
\left(\begin{array}{l}
u(x, t) \\
v(x, t)
\end{array}\right)=\int_{0}^{\infty}\left(\begin{array}{l}
\varphi_{1}(\epsilon) U(x, t, \epsilon) \\
\varphi_{2}(\epsilon) V(x, t, \epsilon)
\end{array}\right) d \epsilon .
$$


Obviously, this solution satisfies the initial condition

$$
\left(\begin{array}{l}
u(x, 0) \\
v(x, 0)
\end{array}\right)=\left(\begin{array}{c}
\Phi_{1}\left(x^{2}\right) \\
\frac{B}{A} \Phi_{2}\left(x^{2}\right)
\end{array}\right),
$$

where $\Phi_{i}$ is the Laplace transform of $\varphi_{i}, i=1,2$. Expressing $U$ and $V$ as Laplace transforms, we can rewrite our solution as

$$
\begin{aligned}
& \left(\begin{array}{l}
u(x, t) \\
v(x, t)
\end{array}\right)=\int_{0}^{\infty} \int_{0}^{\infty}\left(\begin{array}{c}
\varphi_{1}(\epsilon) \frac{\sqrt{x} y^{\mu / 2} e^{-\frac{x^{2}+y}{4 t}} I_{\mu}\left(\frac{x \sqrt{y}}{2 t}\right)}{4 t} \\
\varphi_{2}(\epsilon) \frac{B}{A} \frac{\sqrt{x} y^{\mu / 2} e^{-\frac{x^{2}+y}{4 t}} I_{\mu}\left(\frac{x \sqrt{y}}{2 t}\right)}{4 t}
\end{array}\right) e^{-\epsilon y} d y d \epsilon \\
& =\int_{0}^{\infty}\left(\begin{array}{c}
\Phi_{1}(y) \frac{\sqrt{x} y^{\mu / 2} e^{-\frac{x^{2}+y}{4 t}} I_{\mu}\left(\frac{x \sqrt{y}}{2 t}\right)}{4 t} \\
\Phi_{2}(y) \frac{B}{A} \frac{\sqrt{x} y^{\mu / 2} e^{-\frac{x^{2}+y}{4 t}} I_{\mu}\left(\frac{x \sqrt{y}}{2 t}\right)}{4 t}
\end{array}\right) d y
\end{aligned}
$$

after reversing the order of integration. The change of variables $y \rightarrow y^{2}$ gives, after letting $f(y)=$ $\Phi_{1}\left(y^{2}\right)$ and $g(y)=\Phi_{2}\left(y^{2}\right)$, the solution

$$
\begin{aligned}
\left(\begin{array}{l}
u(x, t) \\
v(x, t)
\end{array}\right) & =\int_{0}^{\infty}\left(\begin{array}{c}
f(y) \frac{\sqrt{x} y^{1+\mu} e^{-\frac{x^{2}+y^{2}}{2 t}} I_{\mu}\left(\frac{x y}{2 t}\right)}{4 t} \\
g(y) \frac{B}{A} \frac{\sqrt{x} y^{1+\mu} e^{-\frac{x^{2}+y^{2}}{4 t}} I_{\mu}\left(\frac{x y}{2 t}\right)}{2 t}
\end{array}\right) d y \\
& =\int_{0}^{\infty}\left(\begin{array}{c}
\frac{\sqrt{x} y^{1+\mu} e^{-\frac{x^{2}+y^{2}}{2 t}} I_{\mu}\left(\frac{x y}{2 t}\right)}{4 t} \\
0 \\
\frac{B}{A} \frac{\sqrt{x} y^{1+\mu} e^{-\frac{x^{2}+y^{2}}{2 t} I_{\mu}\left(\frac{x y}{2 t}\right)}}{4 t}
\end{array}\right)\left(\begin{array}{l}
f(y) \\
g(y)
\end{array}\right) d y .
\end{aligned}
$$

Other linear systems can be treated by this method, but we will not discuss the problem here.

\section{INTEGRAL OPERATORS FROM TRANSLATION AND SCALING SYMMETRIES}

The most basic symmetries and certainly the easiest to find are those arising from translations and scalings. We turn first to translation symmetries. That is, we consider the situation in which the transformation $u\left(x_{1}, \ldots, x_{k}, \ldots, x_{n}\right) \rightarrow u\left(x_{1}, \ldots, x_{k}-\epsilon_{k}, \ldots, x_{n}\right)$ is a symmetry for some linear $\operatorname{PDE} \mathcal{L} u=0$

The method outlined above leads us to the operator

$$
\tilde{u}(x)=\int_{\Omega} \varphi(\epsilon) u\left(x_{1}, \ldots, x_{k}-\epsilon_{k}, \ldots, x_{n}\right) d \epsilon_{k},
$$

where $\varphi$ lies in a suitable function class and $\Omega$ is an appropriate domain of integration. Of course if translation preserves solutions in other variables, we may extend this operator in the obvious way.

An interesting special case arises from the choice $\varphi(\epsilon)=\prod_{i=1}^{n} \frac{1}{\pi \epsilon_{i}}, \Omega=\mathbb{R}^{n}$ and the integral is taken in the principle value sense. This is essentially a Hilbert transform of the solution. The Hilbert 
transform plays a central role in modern harmonic analysis. ${ }^{27,19,20}$ More generally, if $\mathcal{L} u=0$ has translation symmetries in $k$ variables, where the symmetries are generated by $\mathbf{v}_{i}, i \in K$, where $K$ is an index set such that $|K|=k$, then the Hilbert transform

$$
(H u)(x)=\frac{1}{\pi^{k}} P \int_{\mathbb{R}^{k}} \frac{\exp \left(\epsilon_{i} \mathbf{v}_{i}\right) u(x)}{\epsilon_{1} \cdots \epsilon_{k}} d \epsilon
$$

will be a solution whenever $u$ is, provided that the integral converges.

Example 3.1. Consider the heat equation and the solution $u(x, t)=\frac{1}{\sqrt{4 \pi t}} e^{-x^{2} / 4 t}$. Then, taking the Hilbert transform in $x$ gives

$$
(H u)(x, t)=\frac{e^{-\frac{x^{2}}{4 t}} \operatorname{erfi}\left(\frac{x}{2 \sqrt{t}}\right)}{\sqrt{4 \pi t}}
$$

and it is easy to show that this is indeed a solution. Now suppose that we take $u_{2}(x, t)=e^{-t} \sin x$. Taking the Hilbert transform gives

$$
\left(H u_{2}\right)(x, t)=-e^{-t} \cos x,
$$

which is also a solution. In both cases, we used the table of transforms in Ref. 20.

Our focus is on operators of form (1.1). However, there are other types of symmetry operators that can be constructed from a single point symmetry. We will not here attempt to give a detailed theory, but rather will give an example. Suppose that $\phi(\lambda)$ is an analytic function such that

$$
\phi(\lambda)=\sum_{n=0}^{\infty} \frac{1}{n !} \phi^{(n)}(0) \lambda^{n},|\lambda|<M .
$$

Consider the series

$$
U(x, \epsilon)=\sum_{n=0}^{\infty} \frac{1}{n !} \phi^{(n)}(0) \lambda^{n} u(x-n \epsilon) .
$$

We suppose that this series converges and we can take the Fourier transform in $x$. Then, if $\widehat{U}(\xi, \epsilon)=\int_{\mathbb{R}^{n}} U(x, \epsilon) e^{-i x \cdot \xi} d x$, we have

$$
\begin{aligned}
\widehat{U}(\xi, \epsilon) & =\sum_{n=0}^{\infty} \frac{1}{n !} \phi^{(n)}(0) \lambda^{n} e^{-i n \epsilon \xi} \widehat{u}(\xi) \\
& =\phi\left(\lambda e^{-i \epsilon \cdot \xi}\right) \widehat{u}(\xi) .
\end{aligned}
$$

Fourier inversion gives the operator

$$
(A u)(x, \epsilon, \lambda)=\frac{1}{(2 \pi)^{n}} \int_{\mathbb{R}^{n}} \phi\left(\lambda e^{-i \epsilon \cdot \xi}\right) \widehat{u}(\xi) e^{i x \cdot \xi} d \xi .
$$

Essentially, we have obtained a pseudo-differential operator from translation symmetries. This argument is of course completely formal, having ignored all questions of convergence, but one would hope that $A u$ is also a solution of $\mathcal{L} u=0$.

Let us now suppose that the PDE is equivalent to the pseudo-differential equation

$$
(P(D) u)(x)=\frac{1}{(2 \pi)^{n}} \int_{\mathbb{R}^{n}} p(\xi) \widehat{u}(\xi) e^{i x \cdot \xi} d \xi=0 .
$$

We assume the pointwise product $p \widehat{u} \in L^{1}\left(\mathbb{R}^{n}\right)$. If translation is a symmetry, then

$$
\frac{1}{(2 \pi)^{n}} \int_{\mathbb{R}^{n}} p(\xi) \widehat{u}(\xi) e^{i(x-\epsilon) \cdot \xi} d \xi=0
$$


whenever $(P(D) u)(x)=0$. If we denote Fourier transform by $\mathcal{F}$, then $(\mathcal{F} A u)(\eta, \epsilon, \lambda)=\phi\left(\lambda e^{-i \epsilon \cdot \eta}\right)$ $\widehat{u}(\eta)$. Hence,

$$
(P(D) A u)(x, \epsilon, \lambda)=\frac{1}{(2 \pi)^{n}} \int_{\mathbb{R}^{n}} p(\eta) \phi\left(\lambda e^{-i \epsilon \cdot \eta}\right) \widehat{u}(\eta) e^{i x \cdot \eta} d \eta
$$

Let

$$
\psi_{N}(x, \eta, \epsilon, \lambda)=p(\eta) \widehat{u}(\eta) \sum_{k=1}^{N} \frac{\lambda^{n}}{n !} \phi^{(n)}(0) e^{i(x-k \epsilon) \cdot \eta} .
$$

Clearly, $\psi_{N}(x, \eta, \epsilon, \lambda) \rightarrow p(\eta) \phi\left(\lambda e^{-i \epsilon \cdot \eta}\right) \widehat{u}(\eta) e^{i x \cdot \eta}$ pointwise in $\eta$ as $N \rightarrow \infty$. Further,

$$
\left|\psi_{N}(x, \eta, \epsilon, \lambda)\right| \leq|p(\eta) \widehat{u}(\eta)||\phi(|\lambda|)|,
$$

which is integrable in $\eta$. Since translation is a symmetry, it follows that

$$
\frac{1}{(2 \pi)^{n}} \int_{\mathbb{R}^{n}} \psi_{N}(x, \eta, \epsilon, \lambda) d \eta=0 .
$$

By the dominated convergence theorem, we conclude that

$$
(P(D) A u)(x, \epsilon, \lambda)=\lim _{N \rightarrow \infty} \frac{1}{(2 \pi)^{n}} \int_{\mathbb{R}^{n}} \psi_{N}(x, \eta, \epsilon, \lambda) d \eta=0 .
$$

We have thus proved the following.

Theorem 3.1. For $p \widehat{u} \in L^{1}\left(\mathbb{R}^{n}\right)$ let

$$
(P(D) u)(x)=\frac{1}{(2 \pi)^{n}} \int_{\mathbb{R}^{n}} p(\xi) \widehat{u}(\xi) e^{i x \cdot \xi} d \xi .
$$

Suppose that translation in $x$ preserves solutions of the pseudo-differential equation $(P(D) u)(x)=$ 0 . Then if $\phi$ is given by (3.4), the mapping

$$
(A u)(x, \epsilon, \lambda)=\frac{1}{(2 \pi)^{n}} \int_{\mathbb{R}^{n}} \phi\left(\lambda e^{-i \epsilon \cdot \xi}\right) \widehat{u}(\xi) e^{i x \cdot \xi} d \xi
$$

is also a symmetry of $(P(D) u)(x)=0$.

Corollary 3.2. Let $\varphi$ be a compactly supported function, with support in $\Omega$. Then,

$$
U(x, \lambda)=\int_{\Omega} \varphi(\epsilon)(A u)(x, \epsilon, \lambda) d \epsilon
$$

is a solution of

$$
(P(D) u)(x)=\frac{1}{(2 \pi)^{n}} \int_{\mathbb{R}^{n}} p(\xi) \widehat{u}(\xi) e^{i x \cdot \xi} d \xi=0,
$$

whenever $u$ is.

Of course we could also integrate $U(x, \lambda)$ against a test function in $\lambda$ and obtain another transform, but as they stand, these results are somewhat theoretical. It is relatively easy to obtain examples of the operator defined by Theorem 3.1, but more difficult to actually evaluate the resulting solutions explicitly, unless $u$ is itself a trivial solution. But it should be clear that even apparently trivial symmetries may potentially be turned into more complicated integral operators.

\section{A. Fourier analysis with scaling symmetries}

In order for the symmetry group Fourier transform to be useful, we either have to have what we may refer to (rather imprecisely) as an "interesting" symmetry, such as the one used in the earlier 
heat equation example, or a non-trivial solution. For scaling symmetries, we obviously require a non-constant solution. These are often easy to obtain, by an ansatz, separation of variables, or sometimes simply by inspection.

As ever, we start with the one dimensional heat equation. An elementary solution is $u_{1}(x, t)=$ $e^{x+t}$. The heat equation has a scaling symmetry

$$
u(x, t) \rightarrow u\left(\lambda x, \lambda^{2} t\right) .
$$

Applying this to $u_{1}$ gives the solution $\tilde{u}_{1}(x, t, \lambda)=e^{\lambda x+\lambda^{2} t}$. Now let $\lambda \rightarrow i \lambda$. We then have the solution

$$
\tilde{u}_{1}(x, t ; i \lambda)=e^{i \lambda x-\lambda^{2} t}
$$

Now consider the solution

$$
U(x, t ; y)=\int_{-\infty}^{\infty} \varphi(\lambda, y) e^{i \lambda x-\lambda^{2} t} d \lambda .
$$

We have let the test function depend on two variables. This gives us another family of solutions indexed by $y$. Consequently, we can build solutions using the same approach. That is, let

$$
\tilde{U}(x, t)=\int_{-\infty}^{\infty} f(y) U(x, t ; y) d y .
$$

If we take $\varphi(\lambda, y)=\frac{1}{2 \pi} e^{-i \lambda y}$, then we have the solution

$$
\tilde{U}(x, t)=\int_{-\infty}^{\infty} f(y) \frac{1}{\sqrt{4 \pi t}} e^{-\frac{(x-y)^{2}}{4 t}} d y .
$$

Thus, even from a scaling symmetry and a quite trivial solution, we are able to construct the heat kernel with very little work.

Of course the heat equation has a rich symmetry group. To consider an equation which does not have as many useful symmetries, let us study the third order PDE $u_{t}=u_{x x x}$. The only symmetries possessed by this equation are multiplication of the solution by a constant, translations in $x$ and $t$, and scalings. But if we use the scaling symmetry $u(x, t) \rightarrow u\left(\lambda x, \lambda^{3} t\right)$ and the solution $u(x, t)=e^{x+t}$, then we obtain $\tilde{u}(x, t ; \lambda)=e^{\lambda x+\lambda^{3} t}$. Again, we complexify $\lambda$ and obtain the solution

$$
U(x, t)=\int_{-\infty}^{\infty} \phi(\lambda) e^{i \lambda x-i \lambda^{3} t} d \lambda .
$$

So again we can recover the Fourier transform solution-and hence the fundamental solutionfrom a simple solution, a scaling symmetry, and the process of integrating against a test function.

So far, none of this is terribly surprising. We can obtain the solutions of the previous two equations by standard Fourier analysis, but let us generalize the idea. Every scaling symmetry is of the form $u(x, t) \rightarrow \lambda^{\alpha} u\left(\lambda^{\beta}, \lambda^{\gamma} t\right)$, where we take $\beta$ and $\gamma$ to be non-zero. The $\lambda^{\alpha}$ term is irrelevant as it can be absorbed into the test function. Thus, we may consider the solution

$$
U(x, t)=\int_{0}^{\infty} \varphi(\lambda) u\left(\lambda^{\beta}, \lambda^{\gamma} t\right) d \lambda .
$$

The requirement that $U(x, 0)=f(x)$ leads to the integral transform

$$
f(x)=\int_{0}^{\infty} \varphi(\lambda) u\left(\lambda^{\beta}, 0\right) d \lambda .
$$

Obviously, the change of parameter $\lambda \rightarrow \lambda^{1 / \beta}$ leads to the equation

$$
f(x)=\int_{0}^{\infty} \phi(\lambda) u(\lambda x, 0) d \lambda,
$$

where $\phi$ is obtained from $\varphi$ in the obvious way. So without loss of generality, we consider the case $\beta=1$. We assume that $u$ is not time independent. For many choices of $u$, this is a known integral transform. 
More generally, following Titchmarsh (see the material on Mellin type kernels in the book ${ }^{29}$ ), we take the Mellin transform of both sides of (3.18). We are assuming here that $u\left(\lambda x, \lambda^{\gamma} t\right) \neq$ $\lambda^{r} u\left(x, \lambda^{\gamma} t\right)$ for any $r$. We also require that $u$ is not group invariant, or more generally that $u(\lambda x$, $\left.\lambda^{\gamma} t\right) \neq c(\lambda) u(x, t)$ for any $c(\lambda)$.

Recall that the Mellin transform is defined by

$$
(\mathcal{M} f)(s)=\int_{0}^{\infty} f(x) x^{s-1} d x,
$$

for $s \in \Omega \subset \mathbb{C}$. Typically, the transform exists for $s$ in some strip, $s_{1}<\mathfrak{R}(s)<s_{2}$. See Ref. 31 for a discussion of the Mellin transform.

Applying this to (3.18) gives

$$
\begin{aligned}
F(s) & =\int_{0}^{\infty} \int_{0}^{\infty} \phi(\lambda) u(\lambda x, 0) x^{s-1} d x d \lambda \\
& =\int_{0}^{\infty} \int_{0}^{\infty} \phi(\lambda) u(y)\left(\frac{y}{\lambda}\right)^{s-1} \frac{d y}{\lambda} d \lambda \\
& =\Phi(1-s) \bar{u}(s, 0),
\end{aligned}
$$

where $F$ and $\Phi$ are the Mellin transforms of $f$ and $\phi$ and $\bar{u}$ is the Mellin transform of $u$. We therefore have

$$
\Phi(1-s)=\frac{F(s)}{\bar{u}(s, 0)}
$$

Letting $s=1-k$ leads to

$$
\Phi(k)=\frac{F(1-k)}{\bar{u}(1-k, 0)} .
$$

Using the convolution theorem for the Mellin transform, a standard argument (see Ref. 13, p. 352) allows us to write

$$
\begin{aligned}
\phi(\lambda) & =\mathcal{M}^{-1}\left[\frac{F(1-k)}{\bar{u}(1-k, 0)}\right](\lambda) \\
& =\int_{0}^{\infty} f(y) g(\lambda y) d y,
\end{aligned}
$$

where

$$
g(y)=\mathcal{M}^{-1}\left[\frac{1}{\bar{u}(1-k, 0)}\right](y) .
$$

So formally we have the solution of the initial value problem given by

$$
U(x, t)=\int_{0}^{\infty} \int_{0}^{\infty} f(y) g(\lambda y) u\left(\lambda x, \lambda^{\gamma} t\right) d y d \lambda .
$$

We encapsulate this in the following result.

Theorem 3.3. Let $P\left(x, t, \partial_{x}, \partial_{t}\right) u=0$ be a linear PDE which possesses scaling symmetries $u(x, t) \rightarrow u\left(\lambda x, \lambda^{\gamma} t\right)$. Suppose that $u$ is a nontrivial solution, neither homogeneous in $x$ nor invariant under scalings, such that the Mellin transform of $u(x, 0)$ exists in some strip. Then, the problem $P\left(x, t, \partial_{x}, \partial_{t}\right) U=0, U(x, 0)=f(x)$, where $f$ has compact support, has a solution

$$
U(x, t)=\int_{0}^{\infty} \int_{0}^{\infty} f(y) g(\lambda y) u\left(\lambda x, \lambda^{\gamma} t\right) d y d \lambda,
$$

with

$$
g(y)=\mathcal{M}^{-1}\left[\frac{1}{\bar{u}(1-k, 0)}\right](y),
$$

whenever $\frac{1}{\bar{u}(1-k, 0)}$ is itself a Mellin transform. 
Proof. If $f$ has compact support and $g$ exists, then (3.23) converges and differentiation under the integral sign shows that it is a solution. Our previous calculations show that $U(x, 0)=f(x)$.

This result guarantees that the solution generated will satisfy the data $u(x, 0)=f(x)$ but places no constraints on other derivatives. If we have to also satisfy, say, $u_{t}(x, 0)=g(x)$, then further analysis is needed. We will present an example below. Of course one can improve on this result in other ways, for example, by specifying in which function spaces $u$ and $f$ must lie. However, for many functions $u$, it will be possible to invert transform (3.18) immediately. Let us illustrate this situation.

Example 3.2. Suppose we wish to solve the equation

$$
u_{t}=\frac{1}{2} u_{x x}-\frac{A}{x^{2}} u, u(x, 0)=f(x) .
$$

This equation possesses the same scaling symmetries as the heat equation. The simplest way to obtain a non-trivial solution is to look for a separable solution. With separation constant equal to one half, this leads to $u_{1}(x, t)=\sqrt{x} I_{v}(x) e^{-1 / 2 t}$, where $v=\frac{1}{2} \sqrt{8 A+1}$. Applying the symmetry and complexifying as before, we have

$$
u(x, t)=\int_{0}^{\infty} \varphi(\lambda) \sqrt{\lambda x} J_{v}(\lambda x) e^{-\frac{1}{2} \lambda^{2} t} d \lambda .
$$

We absorbed the terms in $i$ into $\varphi$. Setting $u(x, 0)=f(x)$ gives

$$
f(x)=\int_{0}^{\infty} \varphi(\lambda) \sqrt{\lambda x} J_{v}(\lambda x) d \lambda .
$$

So $f$ is the Hankel transform of $\varphi$. The inversion theorem for the Hankel transform yields

$$
\varphi(\lambda)=\int_{0}^{\infty} f(y) \sqrt{\lambda y} J_{v}(\lambda y) d y .
$$

Hence,

$$
\begin{aligned}
u(x, t) & =\int_{0}^{\infty}\left(\mathcal{H}_{v} f\right)(\lambda) \sqrt{\lambda x} J_{v}(\lambda x) e^{-\frac{1}{2} \lambda^{2} t} d \lambda \\
& =\int_{0}^{\infty} \int_{0}^{\infty} f(y) \sqrt{x y} \lambda J_{v}(\lambda x) J_{v}(\lambda y) e^{-\frac{1}{2} \lambda^{2} t} d \lambda d y \\
& =\int_{0}^{\infty} f(y) \frac{\sqrt{x y}}{t} \exp \left(-\frac{x^{2}+y^{2}}{2 t}\right) I_{v}\left(\frac{x y}{t}\right) d y
\end{aligned}
$$

solves our initial value problem. We used formula 6.633(2) on page 699 in Ref. 16 for the final integral. Thus, we have obtained a fundamental solution from a separable solution and a scaling symmetry.

Example 3.3. The fourth order PDE $u_{t}=x^{2} u_{x x x x}, x \geq 0$ has scaling symmetries of the form $u(x, t) \rightarrow \lambda^{\alpha} u\left(\lambda x, \lambda^{2} t\right)$. Observe that separation of variables gives the solution

$$
w(x, t)=x K_{2}(2 \sqrt{x}) e^{t},
$$

where $K_{2}(z)$ is the modified Bessel function of the second kind, see 9.6.2 of Ref. 1 .

We will solve the equation subject to $u(x, 0)=f(x)$, where $f$ has compact support. Letting $\lambda \rightarrow i \lambda$, taking the real part, and using the general procedure lead to the solution

$$
u(x, t)=\int_{0}^{\infty} \varphi(\lambda) x \operatorname{ker}_{2}(2 \sqrt{\lambda x}) e^{-\lambda^{2} t} d \lambda .
$$

Here, $\operatorname{ker}_{2}(z)=\mathfrak{R}\left(e^{-\pi i} K_{2}\left(z e^{i \pi / 4}\right)\right)$ is the Kelvin function given by 9.9.11 of Ref. 1. We note that this function decays very rapidly. We require

$$
\int_{0}^{\infty} \varphi(\lambda) x \operatorname{ker}_{2}(2 \sqrt{\lambda x}) d \lambda=f(x)
$$


We will derive an inversion integral for this transform.

Taking Mellin transforms of both sides of (3.26) gives (see Ref. 14, but the integral can also be done in Mathematica), for $\mathfrak{R}(s)>0$,

$$
\int_{0}^{\infty} \varphi(\lambda) \frac{1}{2} \lambda^{-s-1} \Gamma(s) \Gamma(s+2) \sin \left(\frac{\pi s}{2}\right) d \lambda=F(s) .
$$

Letting $K(s)=\frac{1}{2} \lambda^{-s-1} \Gamma(s) \Gamma(s+2) \sin \left(\frac{\pi s}{2}\right)$, we have a solution to our equation given by

$$
u(x, t)=\int_{0}^{\infty} \int_{0}^{\infty} f(y) k(y \lambda) x \operatorname{ker}_{2}(2 \sqrt{\lambda x}) e^{-\lambda^{2} t} d y d \lambda,
$$

where $k(y)=\mathcal{M}^{-1}\left[\frac{1}{K(1-s)}\right](y)$. Recall that $\Gamma(s) \Gamma(1-s)=\frac{\pi}{\sin (\pi s)}$, see 8.334.3 of Ref. 16. So that

$$
\frac{1}{K(1-s)}=\frac{2 \sec \left(\frac{\pi s}{2}\right)}{\lambda^{s-2} \Gamma(1-s) \Gamma(3-s)}=\frac{4 \lambda^{2} \sin \left(\frac{\pi s}{2}\right) \Gamma(s)}{\pi \lambda^{s} \Gamma(3-s)} .
$$

Since $\frac{1}{\Gamma(s)}$ is an entire function, it follows that $1 / K(1-s)$ is analytic except at $s=0,-1,-2, \ldots$ where there are simple poles. The residues at the poles of the form $-2 n-1$ have the values

$$
\frac{4 \lambda^{2}}{\pi}(-1)^{n} \frac{(\lambda y)^{2 n+1}}{(2 n+1) ! \Gamma(2 n+4)}
$$

and the residues at the negative even integers are zero. The Mellin inversion theorem (see Ref. 26) then gives us

$$
\begin{aligned}
\mathcal{M}^{-1}\left[\frac{1}{K(1-s)}\right] & =\sum_{n=0}^{\infty} \frac{4 \lambda^{2}}{\pi}(-1)^{n} \frac{(\lambda y)^{2 n+1}}{(2 n+1) ! \Gamma(2 n+4)} \\
& =\frac{4 \lambda \operatorname{ber}_{2}(2 \sqrt{\lambda y})}{\pi y} .
\end{aligned}
$$

Here, $\operatorname{ber}_{v}(z)$ is the Kelvin function defined by equation 9.9 .9 of Ref. 1. So our solution is

$$
\begin{aligned}
u(x, t) & =\int_{0}^{\infty} \int_{0}^{\infty} f(y) \frac{4 \lambda \operatorname{ber}_{2}(2 \sqrt{\lambda y})}{\pi y} x \operatorname{ker}_{2}(2 \sqrt{\lambda x}) e^{-\lambda^{2} t} d y d \lambda \\
& =\int_{0}^{\infty} f(y) H(t, x, y) d y,
\end{aligned}
$$

where

$$
H(t, x, y)=\int_{0}^{\infty} \frac{4 \lambda \operatorname{ber}_{2}(2 \sqrt{\lambda y})}{\pi y} x \operatorname{ker}_{2}(2 \sqrt{\lambda x}) e^{-\lambda^{2} t} d \lambda .
$$

The integral defining the kernel $H$ is convergent, since $\operatorname{ber}_{2}(\sqrt{z}) \sim e^{\sqrt{z / 2}} z^{-1 / 4}$ for $z$ large (see 9.10.1 of Ref. 1) and ker 2 decays rapidly. We have not yet evaluated it. It is also interesting to note that as a consequence of this analysis, we have derived an integral transform pair, specifically

$$
\begin{aligned}
& f(x)=\int_{0}^{\infty} \varphi(\lambda) x \operatorname{ker}_{2}(2 \sqrt{\lambda x}) d \lambda, \\
& \varphi(\lambda)=\int_{0}^{\infty} f(x) \frac{4 \lambda \operatorname{ber}_{2}(2 \sqrt{\lambda x})}{\pi x} d x .
\end{aligned}
$$

We will not investigate properties of this transform pair further, but see Ref. 30 for more on this method.

Example 3.4. Consider now the Cauchy problem

$$
u_{t t}=x^{2} u_{x x x x}, u(x, 0)=f(x), u_{t}(x, 0)=g(x), x \geq 0 .
$$

We will once more suppose that $f$ and $g$ have compact support. The PDE possesses scaling symmetries $u(x, t) \rightarrow u(\lambda x, \lambda t)$. We choose two solutions, 


$$
u_{1}(x, t)=x \operatorname{ker}_{2}(2 \sqrt{x}) \cos t \text { and } u_{2}(x, t)=x \operatorname{ker}_{2}(2 \sqrt{x}) \sin t
$$

and build a solution

$$
\begin{aligned}
u(x, t)= & \int_{0}^{\infty} \varphi(\lambda) x \operatorname{ker}_{2}(2 \sqrt{\lambda x}) \cos (\lambda t) d \lambda \\
& +\int_{0}^{\infty} \psi(\lambda) x \operatorname{ker}_{2}(2 \sqrt{\lambda x}) \sin (\lambda t) d \lambda .
\end{aligned}
$$

Now we require

$$
u(x, 0)=\int_{0}^{\infty} \varphi(\lambda) x \operatorname{ker}_{2}(2 \sqrt{\lambda x}) d \lambda=f(x)
$$

and from our previous example, we see that

$$
\varphi(\lambda)=\int_{0}^{\infty} f(x) \frac{4 \lambda \mathrm{ber}_{2}(2 \sqrt{\lambda x})}{\pi x} d x .
$$

Also

$$
u_{t}(x, 0)=\int_{0}^{\infty} \lambda \psi(\lambda) x \operatorname{ker}_{2}(2 \sqrt{\lambda x}) d \lambda=g(x)
$$

so that

$$
\psi(\lambda)=\frac{1}{\lambda} \int_{0}^{\infty} g(x) \frac{4 \lambda \text { ber }_{2}(2 \sqrt{\lambda x})}{\pi x} d x=\int_{0}^{\infty} g(x) \frac{4 \operatorname{ber}_{2}(2 \sqrt{\lambda x})}{\pi x} d x .
$$

So we have a solution to our Cauchy problem given by

$$
\begin{aligned}
u(x, t) & =\int_{0}^{\infty} \int_{0}^{\infty} f(y) \frac{4 \lambda \operatorname{ber}_{2}(2 \sqrt{\lambda y})}{\pi y} x \operatorname{ker}_{2}(2 \sqrt{\lambda x}) \cos (\lambda t) d y d \lambda \\
& +\int_{0}^{\infty} \int_{0}^{\infty} g(y) \frac{4 \operatorname{ber}_{2}(2 \sqrt{\lambda y})}{\pi y} x \operatorname{ker}_{2}(2 \sqrt{\lambda x}) \sin (\lambda t) d y d \lambda .
\end{aligned}
$$

Example 3.5. Let us consider the equation $u_{t}=y\left(u_{x x}+u_{y y}\right)+u_{y}$, where $x \in \mathbb{R}$ and $y, t>0$. This equation possesses translation symmetries in $x$ and $t$ and the scaling symmetry $u(x, y, t) \rightarrow$ $u(\lambda x, \lambda y, \lambda t)$. Separation of variables gives the solution $v(x, y, t)=e^{-y-t} \cos x$. Thus,

$$
U(x, y, t)=\int_{0}^{\infty} \varphi(\lambda) e^{-\lambda(y+t)} \cos (\lambda x) d \lambda
$$

is also a solution. The choice $\varphi(\lambda)=1 / \pi$ produces the solution $K(x, y, t)=\frac{y+t}{\pi\left(x^{2}+(y+t)^{2}\right)}$. Observe that $K(x, y, 0)$ is the Poisson kernel for the upper half-plane. As translation in $x$ is a symmetry, we conclude that for $\phi \in L^{1}(\mathbb{R})$,

$$
u(x, y, t)=\int_{-\infty}^{\infty} \phi(\xi) K(x-\xi, y, t) d \xi
$$

is a solution of the problem

$$
u_{t}=y\left(u_{x x}+u_{y y}\right)+u_{y}
$$

with $u(x, y, 0)$ harmonic in the upper half-plane and $u(x, 0,0)=\phi(x)$. For example, the choice $\phi(\xi)=e^{-\xi^{2}}$ produces the solution

$$
u(x, y, t)=\frac{1}{2}\left(e^{(t-i x+y)^{2}} \operatorname{erfc}(t-i x+y)+e^{(t+i x+y)^{2}} \operatorname{erfc}(t+i x+y)\right) .
$$

It is not surprising that

$$
P \int_{-\infty}^{\infty} \frac{1}{\epsilon} K(x-\epsilon, y, t) d \epsilon=\frac{x}{\pi\left(x^{2}+(y+t)^{2}\right)},
$$

which at $t=0$ gives the conjugate Poisson kernel for the upper half-plane. 
More generally,

$$
w(x, y, t)=\int_{-\infty}^{\infty} \int_{0}^{\infty} \phi(\xi, \lambda) K(\lambda x-\xi, \lambda y, \lambda t) d \lambda d \xi
$$

is also a solution of the PDE. We would like to choose $\phi$ in order that $w(x, y, 0)=f(x, y)$. However, it is not yet clear how to do this.

\section{THE HYPERBOLIC CASE}

Now let us develop the method in the context of second order hyperbolic problems. The natural starting point is the one-dimensional wave equation. We will use our method to derive the D'Alembert solution of the Cauchy problem

$$
u_{t t}=u_{x x}, u(x, 0)=f(x), u_{t}(x, 0)=g(x), x \in \mathbb{R} .
$$

We will proceed by integrating a pair of solutions obtained by the application of Lie symmetries to elementary solutions. It is easily established that if $u_{t t}=u_{x x}$, then

$$
\tilde{u}_{\epsilon}(x, t)=u\left(\frac{x+\epsilon\left(x^{2}-t^{2}\right)}{1+2 \epsilon x+\epsilon^{2}\left(x^{2}-t^{2}\right)}, \frac{t}{1+2 \epsilon x+\epsilon^{2}\left(x^{2}-t^{2}\right)}\right)
$$

also solves the one-dimensional wave equation, see Ref. 3. Since both $x$ and $t$ are solutions, we see that

$$
\begin{aligned}
& k^{1}(x, t ; \epsilon)=\frac{x+\epsilon\left(x^{2}-t^{2}\right)}{1+2 \epsilon x+\epsilon^{2}\left(x^{2}-t^{2}\right)}, \\
& k^{2}(x, t ; \epsilon)=\frac{t}{1+2 \epsilon x+\epsilon^{2}\left(x^{2}-t^{2}\right)}
\end{aligned}
$$

solve the wave equation. As in the fourth order PDE of Example 3.4, we form a solution by integrating these against test functions $\varphi$ and $\psi$, with suitable decay. So we obtain a solution of the wave equation of the form

$$
u(x, t)=\int_{0}^{\infty} \varphi(\epsilon) k^{1}(x, t ; \epsilon) d \epsilon+\int_{0}^{\infty} \psi(\epsilon) k^{2}(x, t ; \epsilon) d \epsilon .
$$

Thus, $u$ is defined in terms of a pair of integral kernel operators, where the kernels are constructed from the symmetries of the equation. To study this, we represent the solutions $k^{1}, k^{2}$ as Laplace transforms, by analogy with the heat equation case. In the first case, we have

$$
\begin{aligned}
k^{1}(x, t ; \epsilon) & =\frac{x+\epsilon\left(x^{2}-t^{2}\right)}{\left(x^{2}-t^{2}\right)\left[\left(x+\frac{x}{x^{2}-t^{2}}\right)^{2}-\frac{t^{2}}{\left(x^{2}-t^{2}\right)^{2}}\right]} \\
& =\frac{1}{2} \int_{0}^{\infty}\left(\exp \left(-\frac{\xi}{t+x}\right)+\exp \left(\frac{\xi}{t-x}\right)\right) \exp (-\epsilon \xi) d \xi .
\end{aligned}
$$

A similar calculation shows that

$$
k^{2}(x, t ; \epsilon)=-\frac{1}{2} \int_{0}^{\infty}\left(\exp \left(\frac{\eta}{t+x}\right)-\exp \left(\frac{-\eta}{t-x}\right)\right) \exp (-\epsilon \eta) d \eta .
$$

Using these identities, we arrive at

$$
\begin{aligned}
u(x, t)= & \frac{1}{2} \int_{\mathbb{R}_{+}^{2}} \varphi(\epsilon)\left(\exp \left(-\frac{\xi}{t+x}\right)+\exp \left(\frac{\xi}{t-x}\right)\right) \exp (-\epsilon \xi) d \xi d \epsilon \\
& -\frac{1}{2} \int_{\mathbb{R}_{+}^{2}} \psi(\delta)\left(\exp \left(\frac{\eta}{t+x}\right)-\exp \left(\frac{-\eta}{t-x}\right)\right) \exp (-\epsilon \eta) d \eta d \delta,
\end{aligned}
$$

in which $\int_{\mathbb{R}_{+}^{2}} f=\int_{0}^{\infty} \int_{0}^{\infty} f$. 
Now let $\Phi$ and $\Psi$ be the Laplace transforms of $\varphi$ and $\psi$, respectively. Observe that when we reverse the order of integration, we arrive at

$$
u(x, t)=\frac{1}{2} \int_{0}^{\infty} \Phi(\xi)\left(e^{-\frac{\xi}{t+x}}+e^{\frac{\xi}{t-x}}\right) d \xi-\frac{1}{2} \int_{0}^{\infty} \Psi(\eta)\left(e^{\frac{\eta}{t+x}}-e^{\frac{\eta}{t-x}}\right) d \eta,
$$

leading to

$$
u(x, 0)=\int_{0}^{\infty} \Phi(\xi) \exp \left(-\frac{\xi}{x}\right) d \xi=\widehat{\Phi}\left(\frac{1}{x}\right)
$$

and

$$
u_{t}(x, 0)=\frac{1}{x^{2}} \int_{0}^{\infty} \eta \Psi(\xi) \exp \left(-\frac{\eta}{x}\right) d \eta=\frac{d}{d x} \widehat{\Psi}\left(\frac{1}{x}\right) .
$$

Here, $\widehat{\Phi}$ and $\widehat{\Psi}$ denote the Laplace transforms of $\Phi$ and $\Psi$. To solve our initial value problem, we must choose $\Phi$ and $\Psi$ so that

$$
\widehat{\Phi}\left(\frac{1}{x}\right)=f(x) \text { and } \frac{d}{d x} \widehat{\Psi}\left(\frac{1}{x}\right)=g(x) .
$$

Denoting Laplace transform by $\mathcal{L}$, we require

$$
\Phi(\xi)=\mathcal{L}^{-1}(\tilde{f})(\xi),
$$

where $\tilde{f}(x)=f(1 / x)$. We also note that if $\delta_{a}$ is the Dirac delta function at $a$ then $\mathcal{L}\left(\delta_{a}\right)(\xi)=$ $\exp (-a \xi)$. Recall also that

$$
\int_{0}^{\infty} \mathcal{L}(f)(\xi) \mathcal{L}^{-1}(g)(\xi) d \xi=\int_{0}^{\infty} f(\xi) g(\xi) d \xi .
$$

It therefore follows that

$$
\begin{aligned}
\frac{1}{2} \int_{0}^{\infty} \Phi(\xi)\left(e^{-\frac{\xi}{t+x}}+\right. & \left.e^{\frac{\xi}{t-x}}\right) d \xi=\frac{1}{2} \int_{0}^{\infty} \mathcal{L}^{-1}(\tilde{f})(\xi) \mathcal{L}\left(\delta_{\frac{1}{x-t}}+\delta_{\frac{1}{x+t}}\right)(\xi) d \xi \\
& =\frac{1}{2} \int_{0}^{\infty} \tilde{f}(\xi)\left(\delta\left(\xi-\frac{1}{x-t}\right)+\delta\left(\xi-\frac{1}{x+t}\right)\right) d \xi \\
& =\frac{1}{2}\left[\tilde{f}\left(\frac{1}{x-t}\right)+\tilde{f}\left(\frac{1}{x+t}\right)\right] \\
& =\frac{1}{2}[f(x-t)+f(x+t)] .
\end{aligned}
$$

Since $\frac{d}{d x} \widehat{\Psi}\left(\frac{1}{x}\right)=g(x)$, we can write

$$
\widehat{\Psi}(\eta)=\int_{-\infty}^{\frac{1}{\eta}} g(s) d s=G(\eta)
$$

and so $\Psi(x)=\mathcal{L}^{-1}(G)(x)$. We now write

$$
\begin{aligned}
\frac{1}{2} \int_{0}^{\infty} \Psi(\eta)\left(e^{\frac{\eta}{t+x}}-e^{\frac{\eta}{t-x}}\right) d \eta & =\frac{1}{2} \int_{0}^{\infty} \mathcal{L}^{-1}(G)(\eta) \mathcal{L}\left(\delta_{\frac{1}{x-t}}-\delta_{\frac{1}{x+t}}\right)(\eta) d \eta \\
& =\frac{1}{2}\left(G\left(\frac{1}{x-t}\right)-G\left(\frac{1}{x+t}\right)\right) \\
& =\frac{1}{2}\left(\int_{-\infty}^{x-t} g(\eta) d \eta-\int_{-\infty}^{x+t} g(\eta) d \eta\right) \\
& =-\frac{1}{2} \int_{x-t}^{x+t} g(\eta) d \eta .
\end{aligned}
$$

The solution of the wave equation satisfying the given initial data is therefore 


$$
u(x, t)=\frac{1}{2}[f(x-t)+f(x+t)]+\frac{1}{2} \int_{x-t}^{x+t} g(\eta) d \eta .
$$

We have thus recovered the D'Alembert solution of the one-dimensional wave equation.

\section{WAVE EQUATIONS WITH POTENTIAL}

Many of the analytical issues that arise with this method are encapsulated in the equation

$$
u_{t t}=u_{x x}-\frac{1}{A^{2} x^{2}} u, 0 \leq x \leq t, A \neq 0 .
$$

We will give a detailed analysis of this problem.

The Lie symmetry algebra is spanned by $\mathbf{v}_{1}=\partial_{t}, \mathbf{v}_{2}=x \partial_{x}+t \partial_{t}, \mathbf{v}_{3}=2 x t \partial_{x}+\left(x^{2}+t^{2}\right) \partial_{t}, \mathbf{v}_{4}=$ $u \partial_{u}$ (as usual, $\partial_{u}=\partial / \partial u$ etc). The symmetry group is isomorphic to $S L(2, \mathbb{R}) \times \mathbb{R}$. We introduce the constant $\mu=\frac{\sqrt{4+A^{2}}}{2 A}$. We may easily show then that if we set

$$
K(x, t ; \epsilon)=\frac{x^{\mu+\frac{1}{2}}}{\left(1+2 \epsilon t+\epsilon^{2}\left(t^{2}-x^{2}\right)\right)^{\mu+\frac{1}{2}}},
$$

then for $\varphi$ with suitable decay,

$$
u(x, t)=\int_{0}^{\infty} \varphi(\epsilon) K(x, t ; \epsilon) d \epsilon
$$

is a solution of (5.1). Here, we have taken $u_{0}(x, t)=x^{\mu+1 / 2}$ and applied the symmetry arising from $\mathbf{v}_{3}$. For $x<t$, it is easy to see that the singular points of the kernel are $\epsilon=-\frac{1}{t+x}$ and $\epsilon=-\frac{1}{t-x}$ which are to the left of the region of integration, so that integral (5.3) is convergent and defines solutions of the PDE. For $x>t$, the singularities are on the positive axis, so we could perform the analysis by taking the range of integration over the negative $\epsilon$ axis.

We will rewrite this using the Laplace transform to obtain two integral kernel operators. Elementary algebra shows that

$$
u(x, t)=\left(\frac{x}{t^{2}-x^{2}}\right)^{\mu+\frac{1}{2}} \int_{0}^{\infty} \varphi(\epsilon) G(x, t, \epsilon) d \epsilon,
$$

where

$$
G(x, t, \epsilon)=\left(\left(\epsilon+\frac{t}{t^{2}-x^{2}}\right)^{2}-\frac{x^{2}}{\left(t^{2}-x^{2}\right)^{2}}\right)^{-\mu-\frac{1}{2}} .
$$

We use the standard Laplace transform identity (see Ref. 14)

$$
\frac{1}{\left((\epsilon+\alpha)^{2}-\beta^{2}\right)^{v}}=\frac{\sqrt{\pi}}{(2 \beta)^{\nu-1 / 2} \Gamma(v)} \int_{0}^{\infty} \xi^{\nu-1 / 2} I_{\nu-1 / 2}(\beta \xi) e^{-(\epsilon+\alpha) \xi} d \xi,
$$

where $I_{v}$ is the usual modified Bessel function and $\Gamma$ is the gamma function, see Ref. 22. Using this, we are able to write

$$
u(x, t)=c_{\mu} \sqrt{\frac{x}{t^{2}-x^{2}}} \int_{\mathbb{R}_{+}^{2}} \varphi(\epsilon) \xi^{\mu} \exp \left(\frac{-t \xi}{t^{2}-x^{2}}-\epsilon \xi\right) I_{\mu}\left(\frac{x \xi}{t^{2}-x^{2}}\right) d \xi d \epsilon,
$$

where $c_{\mu}=2^{-\mu} \sqrt{\pi} / \Gamma\left(\mu+\frac{1}{2}\right)$. Assuming that $\varphi$ has suitable decay, we can reverse the order of integration and this leads to

$$
\begin{aligned}
u(x, t) & =c_{\mu} \sqrt{\frac{x}{t^{2}-x^{2}}} \int_{0}^{\infty} \Phi(\xi) \xi^{\mu} \exp \left(\frac{-t \xi}{t^{2}-x^{2}}\right) I_{\mu}\left(\frac{x \xi}{t^{2}-x^{2}}\right) d \xi \\
& =\frac{c_{\mu}}{\sqrt{t^{2}-x^{2}}} \int_{0}^{\infty} \Phi(\xi) \xi^{\mu-1 / 2} \sqrt{x \xi} \exp \left(\frac{-t \xi}{t^{2}-x^{2}}\right) I_{\mu}\left(\frac{x \xi}{t^{2}-x^{2}}\right) d \xi .
\end{aligned}
$$

As $\Phi$ is arbitrary, we can absorb $c_{\mu}$ and $\xi^{\mu-1 / 2}$ into it to obtain the more elegant expression 


$$
u(x, t)=\frac{1}{\sqrt{t^{2}-x^{2}}} \int_{0}^{\infty} \Phi(\xi) \sqrt{x \xi} \exp \left(\frac{-t \xi}{t^{2}-x^{2}}\right) I_{\mu}\left(\frac{x \xi}{t^{2}-x^{2}}\right) d \xi
$$

This integral will certainly converge if, for example, $\Phi \in \mathcal{D}\left(\mathbb{R}^{+}\right)$, the compactly supported smooth functions on the positive half-line.

Proposition 5.1. If $\Phi \in \mathcal{D}\left(\mathbb{R}^{+}\right)$, then Equation (5.1) has a solution

$$
u(x, t)=\frac{1}{\sqrt{t^{2}-x^{2}}} \int_{0}^{\infty} \Phi(\xi) \sqrt{x \xi} \exp \left(\frac{-t \xi}{t^{2}-x^{2}}\right) I_{\mu}\left(\frac{x \xi}{t^{2}-x^{2}}\right) d \xi,
$$

satisfying $u(0, t)=0$.

Proof. The condition $u(0, t)=0$ is obvious. The compact support of $\Phi$ guarantees convergence of the integral. Differentiating under the integral sign proves the result.

Actually the integral will also converge for $\Phi \in L^{1}\left(\mathbb{R}^{+}\right)$since using standard estimates for Bessel functions (e.g., Ref. 1, p. 377) for large $\xi$ and $x<t$,

$$
\exp \left(\frac{-t \xi}{t^{2}-x^{2}}\right) I_{\mu}\left(\frac{x \xi}{t^{2}-x^{2}}\right) \sim \frac{\sqrt{t^{2}-x^{2}}}{\sqrt{2 \pi x \xi}} \exp \left(\frac{-\xi}{t+x}\right),
$$

which is enough to guarantee the convergence of the integral in Proposition 5.1. Indeed, the integral will converge for $\Phi$ a polynomial. For example, $\Phi(\xi)=\xi$ gives the solution

$$
u(x, t)=\frac{\Gamma\left(\mu+\frac{5}{2}\right)}{2^{\mu} \Gamma(\mu+1)}\left(t^{2}-x^{2}\right)^{2} \frac{x^{\mu+\frac{1}{2}}}{t^{\mu+\frac{5}{2}}}{ }_{2} F_{1}\left(\frac{5}{4}+\frac{\mu}{2}, \frac{7}{4}+\frac{\mu}{2} ; 1+\mu, \frac{x^{2}}{t^{2}}\right),
$$

where ${ }_{2} F_{1}$ is Gauss' hypergeometric function. ${ }^{22}$

To solve the appropriate Cauchy problem, we require two operators. To this end, we complexify the parameter $\xi$. That is, we replace $\xi$ with $i \xi$ to obtain the solution $v(x, t ; \xi)=\frac{1}{\sqrt{t^{2}-x^{2}}} \sqrt{x \xi}$ $\exp \left(\frac{-i t \xi}{t^{2}-x^{2}}\right) J_{\mu}\left(\frac{x \xi}{t^{2}-x^{2}}\right)$. This leads to the solution

$$
u(x, t)=\frac{1}{\sqrt{t^{2}-x^{2}}} \int_{0}^{\infty} \tilde{\Phi}(\xi) \sqrt{x \xi} \exp \left(\frac{-i t \xi}{t^{2}-x^{2}}\right) J_{\mu}\left(\frac{x \xi}{t^{2}-x^{2}}\right) d \xi .
$$

Here, $J_{\mu}$ is the Bessel function of the first kind. ${ }^{22}$ Since the real and imaginary parts must satisfy the PDE, we obtain a useful corollary. We let $\overline{\mathfrak{H}}\left(\mathbb{R}^{+}\right)$denote the smooth functions $\Phi$ on $\mathbb{R}^{+}$with rapid decrease. That is, $D^{k} \Phi(x)$ (where $D=\frac{d}{d x}$ ) tends to zero faster than any power of $1 / x$ for all $k \in \mathbb{N}$.

Corollary 5.2. If $\Phi, \Psi \in \overline{\mathfrak{H}}\left(\mathbb{R}^{+}\right)$, then

$$
u_{1}(x, t)=\frac{1}{\sqrt{x^{2}-t^{2}}} \int_{0}^{\infty} \Phi(\xi) \sqrt{x \xi} \cos \left(\frac{t \xi}{x^{2}-t^{2}}\right) J_{\mu}\left(\frac{x \xi}{x^{2}-t^{2}}\right) d \xi
$$

and

$$
u_{2}(x, t)=\frac{1}{\sqrt{x^{2}-t^{2}}} \int_{0}^{\infty} \Psi(\xi) \sqrt{x \xi} \sin \left(\frac{t \xi}{x^{2}-t^{2}}\right) J_{\mu}\left(\frac{x \xi}{x^{2}-t^{2}}\right) d \xi
$$

are both solutions of (5.1).

Proof. This is an exercise in differentiation. The integrals are convergent by the rapid decay of $\Phi$ and $\Psi$. We used linearity to replace $t^{2}-x^{2}$ with the more convenient $x^{2}-t^{2}$.

We would like to choose $\Phi$ and $\Psi$ in such a way as to guarantee that $u(x, 0)=f(x)$ and $\frac{\partial u_{2}}{\partial t}(x, 0)=g(x)$ for appropriate initial data $f$ and $g$. The similarity of the operators to the Hankel transform suggests a way of doing this.

Observe that $u_{2}(x, 0)=0$ and $\frac{\partial u_{1}}{\partial t}(x, 0)=0$. In fact, 


$$
\begin{aligned}
u_{1}(x, 0) & =\int_{0}^{\infty} \Phi(\xi) \sqrt{\frac{\xi}{x}} J_{\mu}\left(\frac{\xi}{x}\right) d \xi \\
& =\int_{0}^{\infty} \Phi(\xi) \sqrt{\frac{\xi}{x}} J_{\mu}\left(\frac{\xi}{x}\right) d \xi \\
& =\left(\mathcal{H}_{\mu} \Phi\right)\left(\frac{1}{x}\right),
\end{aligned}
$$

where $\mathcal{H}_{\mu}$ denotes the Hankel transform

$$
\left(\mathcal{H}_{\mu} f\right)(y)=\int_{0}^{\infty} f(x) \sqrt{x y} J_{\mu}(x y) d x .
$$

To satisfy the initial condition $u_{1}(x, 0)=f(x)$, we have to have

$$
\left(\mathcal{H}_{\mu} \Phi\right)\left(\frac{1}{x}\right)=f(x)
$$

which implies

$$
\left(\mathcal{H}_{\mu} \Phi\right)(x)=f^{\sharp}(x)=f(1 / x), x>0 .
$$

As the Hankel transform is its own inverse (see Ref. 32), we have, at least formally,

$$
\Phi(x)=\left(\mathcal{H}_{\mu} f^{\sharp}\right)(x) .
$$

To obtain sufficient conditions on $f$ to justify this analysis, we recall that Zemanian constructed a Frechet space $\mathfrak{S}_{\mu}\left(\mathbb{R}^{+}\right)$characterized by three properties: every $\phi \in \mathfrak{S}_{\mu}\left(\mathbb{R}^{+}\right)$is a rapidly decreasing smooth function on $\mathbb{R}^{+}$, with an expansion of the form

$$
\phi(x)=x^{\mu+1 / 2}\left(a_{0}+a_{2} x^{2}+\cdots+a_{2 k} x^{2 k}+R_{2 k}(x)\right),
$$

where

$$
a_{2 k}=\frac{1}{k ! 2^{k}} \lim _{x \rightarrow 0}\left(x^{-1} D\right)^{k}\left(x^{-\mu-1 / 2} \phi(x)\right)
$$

and the remainder satisfies $\left(x^{-1} D\right)^{k} R_{2 k}(x)=o(1)$ as $x \rightarrow 0^{+}$. (See Lemma 5.2.1 of Ref. 32.) Zemanian proves that the Hankel transform is an automorphism on $\mathfrak{S}_{\mu}\left(\mathbb{R}^{+}\right) \cdot{ }^{32}$ It plays essentially the same role that Schwartz space does for the Fourier transform. Thus, if $f$ is such that $f^{\sharp} \in \mathfrak{G}_{\mu}\left(\mathbb{R}^{+}\right)$, then $\Phi$ will also have rapid decay.

So if $f^{\sharp} \in \mathfrak{S}_{\mu}\left(\mathbb{R}^{+}\right)$, we have a solution of the initial value problem

$$
\begin{aligned}
u_{t t} & =u_{x x}-\frac{1}{(A x)^{2}} u, 0<x<t, \\
u(x, 0) & =f(x), \\
u_{t}(x, 0) & =0
\end{aligned}
$$

given by

$$
u(x, t)=\frac{1}{\sqrt{x^{2}-t^{2}}} \int_{0}^{\infty}\left(\mathcal{H}_{\mu} f^{\sharp}\right)(\xi) \sqrt{x \xi} \cos \left(\frac{t \xi}{x^{2}-t^{2}}\right) J_{\mu}\left(\frac{x \xi}{x^{2}-t^{2}}\right) d \xi .
$$

Turning to the second solution, we find

$$
\left.\frac{\partial}{\partial t} u_{2}(x, t)\right|_{t=0}=\frac{1}{x^{3 / 2}} \int_{0}^{\infty} \xi \Psi(\xi) \sqrt{\frac{\xi}{x}} J_{\mu}\left(\frac{\xi}{x}\right) d \xi
$$

Consequently if $\left.\frac{\partial}{\partial t} u_{2}(x, t)\right|_{t=0}=g(x)$, then

$$
\xi \Psi(\xi)=\left(\mathcal{H}_{\mu} g^{\natural}\right)(\xi),
$$

where $g^{\natural}(\xi)=\xi^{-3 / 2} g(1 / \xi)$. 
So that if $g^{\natural} \in \mathfrak{H}_{\mu}\left(\mathbb{R}^{+}\right)$, this gives us the solution of the initial value problem

$$
\begin{gathered}
u_{t t}=u_{x x}-\frac{1}{(A x)^{2}} u, 0<x<t, \\
u(x, 0)=0, \\
u_{t}(x, 0)=g(x), \\
u(x, t)=\frac{1}{\sqrt{x^{2}-t^{2}}} \int_{0}^{\infty}\left(\mathcal{H}_{\mu} g^{\natural}\right)(\xi) \sqrt{x \xi} \sin \left(\frac{t \xi}{x^{2}-t^{2}}\right) J_{\mu}\left(\frac{x \xi}{x^{2}-t^{2}}\right) \frac{d \xi}{\xi} .
\end{gathered}
$$

This leads to the following result.

Theorem 5.3. The solution of the initial value problem

$$
\begin{aligned}
u_{t t} & =u_{x x}-\frac{1}{(A x)^{2}} u, A \neq 0,0<x<t, \\
u(x, 0) & =f(x)), \\
u_{t}(x, 0) & =g(x),
\end{aligned}
$$

where $f^{\sharp}, g^{\natural} \in \mathfrak{H}_{\mu}\left(\mathbb{R}^{+}\right)$is given by

$$
\begin{aligned}
u(x, t) & =\frac{1}{\sqrt{x^{2}-t^{2}}} \int_{0}^{\infty}\left(\mathcal{H}_{\mu} f^{\sharp}\right)(\xi) \sqrt{x \xi} \cos \left(\frac{t \xi}{x^{2}-t^{2}}\right) J_{\mu}\left(\frac{x \xi}{x^{2}-t^{2}}\right) d \xi \\
& +\frac{1}{\sqrt{x^{2}-t^{2}}} \int_{0}^{\infty}\left(\mathcal{H}_{\mu} g^{\natural}\right)(\xi) \sqrt{x \xi} \sin \left(\frac{t \xi}{x^{2}-t^{2}}\right) J_{\mu}\left(\frac{x \xi}{x^{2}-t^{2}}\right) \frac{d \xi}{\xi} .
\end{aligned}
$$

For the first term in (5.12), we need to evaluate

$$
\int_{0}^{\infty} \int_{0}^{\infty} f^{\sharp}(y) \sqrt{y} \xi \cos \left(\frac{t \xi}{x^{2}-t^{2}}\right) J_{\mu}\left(\frac{x \xi}{x^{2}-t^{2}}\right) J_{\mu}(y \xi) d y d \xi .
$$

Formally, reversing the order of integration leads us to consider integrals of the form

$$
\int_{0}^{\infty} x \cos (c x) J_{\mu}(a x) J_{\mu}(b x) d x
$$

Unfortunately, this integral is divergent in the classical sense. We therefore seek to define it as a distribution. We make use of the result

$$
\begin{array}{rlr}
\int_{0}^{\infty} & \sin (c x) J_{\mu}(a x) J_{\mu}(b x) d x & 0<c<b-a, 0<a<b \\
& =0, \\
& =\frac{1}{2 \sqrt{a b}} P_{\mu-1 / 2}\left(\frac{b^{2}+a^{2}-c^{2}}{2 a b}\right), \quad b-a<c<b+a, 0<a<b \\
& =\frac{\cos (\mu \pi)}{\pi \sqrt{a b}} Q_{\mu-1 / 2}\left(-\frac{b^{2}+a^{2}-c^{2}}{2 a b}\right), b+a<c, 0<a<b,
\end{array}
$$

which is valid for $\mu>-1$. (See formula 6.672.1 of Ref. 16.) Here, $P_{v}$ and $Q_{v}$ are Legendre functions. ${ }^{22}$ These are smooth functions and so their derivatives exist with respect to $c$.

Let $\mathbb{I}_{[a, b]}$ be the indicator function of the interval $[a, b]$. Both the integrals

$$
\begin{aligned}
& \Lambda_{c}^{n}=\int_{0}^{\infty} \mathbb{I}_{[0, n]}(x) x \cos (c x) J_{\mu}(a x) J_{\mu}(b x) d x, \\
& \Xi_{c}^{n}=\int_{0}^{\infty} \mathbb{I}_{[0, n]}(x) \sin (c x) J_{\mu}(a x) J_{\mu}(b x) d x
\end{aligned}
$$


are convergent for finite $n$ and differentiating under the integral sign shows that for each $n \in \mathbb{N}$, $\Lambda_{c}^{n}=\frac{\partial}{\partial c} \Xi_{c}$. Thus, for every test function $\phi \in \mathfrak{H}_{\mu}$, we have

$$
\left(\Lambda_{c}, \phi\right)=\left(\frac{\partial}{\partial c} \Xi_{c}^{n}, \phi\right) .
$$

By the smoothness of the Legendre functions, the $\lim _{n \rightarrow \infty}\left(\frac{\partial}{\partial c} \Xi_{c}^{n}, \phi\right)$ exists and is well defined for all $a, b, c$, and $\phi \in \mathfrak{S}_{\mu}$. So that we may set

$$
\lim _{n \rightarrow \infty}\left(\Lambda_{c}^{n}, \phi\right)=\lim _{n \rightarrow \infty}\left(\frac{\partial}{\partial c} \Xi_{c}^{n}, \phi\right) .
$$

This is equivalent to defining (5.13) by setting

$$
\begin{aligned}
\left(\int_{0}^{\infty} x \cos (c x)\right. & \left.J_{\mu}(a x) J_{\mu}(b x) d x, \phi\right) \\
& =\left(\frac{\partial}{\partial c} \int_{0}^{\infty} \sin (c x) J_{\mu}(a x) J_{\mu}(b x) d x, \phi\right),
\end{aligned}
$$

for all $\phi \in \mathfrak{S}_{\mu}$. We therefore define

$$
\Theta_{\mu}(x, t ; y)=\frac{\sqrt{y}}{\sqrt{x^{2}-t^{2}}} \int_{0}^{\infty} \xi \cos \left(\frac{t \xi}{x^{2}-t^{2}}\right) J_{\mu}(y \xi) J_{\mu}\left(\frac{x \xi}{x^{2}-t^{2}}\right) d \xi
$$

as a distribution via (5.14).

For the second term in (5.17), the desired integral is actually convergent and we set

$$
\Upsilon_{\mu}(x, t ; y)=\frac{1}{\sqrt{x^{2}-t^{2}}} \int_{0}^{\infty} \sin \left(\frac{t \xi}{x^{2}-t^{2}}\right) J_{\mu}(y \xi) J_{\mu}\left(\frac{x \xi}{x^{2}-t^{2}}\right) d \xi .
$$

These provide us with fundamental solutions of our wave equation.

Theorem 5.4. The solution of the initial value problem

$$
\begin{aligned}
u_{t t} & =u_{x x}-\frac{1}{(A x)^{2}} u, A \neq 0,0<x<t, \\
u(x, 0) & =f(x)), \\
u_{t}(x, 0) & =g(x),
\end{aligned}
$$

where $f^{\sharp}, g^{\natural} \in \mathfrak{H}_{\mu}\left(\mathbb{R}^{+}\right)$is given by

$$
u(x, t)=\left(\Theta_{\mu}, f^{\sharp}\right)(x, t)+\left(\Upsilon_{\mu}, g^{\natural}\right)(x, t) .
$$

So once again we are able to explicitly construct the solution of a Cauchy problem by means of Fourier type operates on the symmetry group.

Equations of the form

$$
u_{t t}=u_{x x}+\frac{1}{\left(A e^{\omega x}+B e^{-\omega x}\right)^{2}} u, \omega \in \mathbb{C}
$$

also have non-trivial symmetries. In fact the Lie symmetry group is $S L(2, \mathbb{R}) \times \mathbb{R}$. Analysis of the type just presented is also possible. However, the details are rather more complicated, involving integral transforms with hypergeometric functions in the kernel. We will therefore leave discussion of these equations for another publication.

\section{EQUATIONS WITH VARIABLE WAVE VELOCITY}

In Ref. 3, Bluman and Kumei consider the problem of classifying equations of the form $u_{t t}=c^{2}(x) u_{x x}$ which have non-trivial symmetries. We will not consider every class of equation they treat, but will instead restrict our attention to some illustrative examples. First, we consider 
$c(x)=A e^{B x}$. Actually, we study the equation

$$
u_{t t}=e^{2 x} u_{x x}
$$

The exponential wave speed is perhaps physically unrealistic, but it is nevertheless mathematically interesting. The case of arbitrary $A, B$ follows by a change of variables. This has Lie symmetry algebra spanned by

$$
\mathbf{v}_{1}=\partial_{t}, \mathbf{v}_{2}=\partial_{x}-t \partial_{t}+\frac{1}{2} u \partial_{u}, \mathbf{v}_{3}=t \partial_{x}-\frac{1}{2}\left(t^{2}+e^{-2 x}\right) \partial_{t}+\frac{1}{2} t u \partial_{u} .
$$

Exponentiating $\mathbf{v}_{3}$, we find that if $u$ is a solution of (6.1), then so is

$$
\begin{aligned}
\tilde{u}(x, t ; \epsilon)= & \frac{2 e^{x}}{\sqrt{4 e^{2 x}+4 e^{2 x} \epsilon t+\epsilon^{2}\left(e^{2 x} t^{2}-1\right)}} \times \\
& u\left(\ln \left(e^{x}+\epsilon e^{x} t+\frac{1}{4} \epsilon^{2}\left(e^{x} t^{2}-e^{-x}\right)\right), \frac{2 t e^{2 x}(t \epsilon+2)-2 \epsilon}{e^{2 x}(t \epsilon+2)^{2}-\epsilon^{2}}\right) .
\end{aligned}
$$

We take $u=1$, apply the symmetry, and construct the solution

$$
u(x, t)=\int_{0}^{\infty} \frac{2 \varphi(\epsilon) e^{x}}{\sqrt{4 e^{2 x}+4 e^{2 x} \epsilon t+\epsilon^{2}\left(e^{2 x} t^{2}-1\right)}} d \epsilon
$$

for suitable choice of $\varphi$. Rewriting this using the Laplace transform, exactly as in the two preceding examples, we have

$$
u(x, t)=\frac{2 e^{x}}{\sqrt{e^{2 x} t^{2}-1}} \int_{0}^{\infty} \Phi(\xi) \exp \left(\frac{-2 e^{2 x} t \xi}{e^{2 x} t^{2}-1}\right) I_{0}\left(\frac{2 e^{x} \xi}{e^{2 x} t^{2}-1}\right) d \xi .
$$

Complexifying $\xi$ produces the pair of solutions

$$
\begin{aligned}
& u(x, t)=\frac{2 e^{x}}{\sqrt{e^{2 x} t^{2}-1}} \int_{0}^{\infty} \Phi(\xi) \cos \left(\frac{2 e^{2 x} t \xi}{e^{2 x} t^{2}-1}\right) J_{0}\left(\frac{2 e^{x} \xi}{e^{2 x} t^{2}-1}\right) d \xi, \\
& v(x, t)=\frac{2 e^{x}}{\sqrt{e^{2 x} t^{2}-1}} \int_{0}^{\infty} \Psi(\xi) \sin \left(\frac{2 e^{2 x} t \xi}{e^{2 x} t^{2}-1}\right) J_{0}\left(\frac{2 e^{x} \xi}{e^{2 x} t^{2}-1}\right) d \xi .
\end{aligned}
$$

Again, these are essentially Hankel transforms of $\Psi$ and $\Phi$. Since $v(x, 0)=0$ and $u_{t}(x, 0)=0$, we may solve the initial value problem

$$
\begin{aligned}
w_{t t} & =e^{2 x} w_{x x}, \\
w(x, 0) & =f(x), w_{t}(x, 0)=g(x) .
\end{aligned}
$$

We have $u(x, 0)=-i 2 e^{x}\left(\mathcal{H}_{0} \Phi\right)\left(2 e^{x}\right)$, where for convenience, we use the well known variant of the Hankel transform

$$
\left(\mathcal{H}_{0} f\right)(y)=\int_{0}^{\infty} y f(x) J_{0}(x y) d x .
$$

This is its own inverse. ${ }^{26}$ So if we let $f^{b}(x)=x^{-1} f(\ln (x / 2))$ then the condition $u(x, 0)=f(x)$ implies that $\Phi(y)=i\left(\mathcal{H}_{0} f^{b}\right)(y)$.

Next, $\frac{\partial}{\partial t} u(x, 0)=g(x)$ implies that

$$
\int_{0}^{\infty} \xi \Psi(\xi) J_{0}\left(2 e^{x} \xi\right) d x=-\frac{i}{4} e^{-3 x} g(x) .
$$

Letting $g^{\dagger}(y)=2 / y^{3} g(\ln (y / 2))$, we are lead to the following result.

Theorem 6.1. Let

$$
\Theta(x, t ; y)=\frac{2 i e^{x}}{\sqrt{e^{2 x} t^{2}-1}} \int_{0}^{\infty} \xi \cos \left(\frac{2 e^{2 x} t \xi}{e^{2 x} t^{2}-1}\right) J_{0}\left(\frac{2 e^{x} \xi}{e^{2 x} t^{2}-1}\right) J_{0}(\xi y) d \xi
$$


be defined as a distribution by (5.14) and let

$$
\Upsilon(x, t ; y)=\frac{2 i e^{x}}{\sqrt{e^{2 x} t^{2}-1}} \int_{0}^{\infty} \sin \left(\frac{2 e^{2 x} t \xi}{e^{2 x} t^{2}-1}\right) J_{0}\left(\frac{2 e^{x} \xi}{e^{2 x} t^{2}-1}\right) J_{0}(\xi y) d \xi .
$$

Then, the initial value problem

$$
\begin{aligned}
u_{t t} & =e^{2 x} u_{x x}, \\
u(x, 0) & =f(x), u_{t}(x, 0)=g(x),
\end{aligned}
$$

where $f^{b}, g^{\dagger} \in \mathfrak{H}_{0}\left(\mathbb{R}^{+}\right)$has a solution given by

$$
\begin{aligned}
u(x, t) & =\frac{2 i e^{x}}{\sqrt{e^{2 x} t^{2}-1}} \int_{0}^{\infty}\left(\mathcal{H}_{0} f^{b}\right)(\xi) \cos \left(\frac{2 e^{2 x} t \xi}{e^{2 x} t^{2}-1}\right) J_{0}\left(\frac{2 e^{x} \xi}{e^{2 x} t^{2}-1}\right) d \xi \\
& -\frac{2 i e^{x}}{\sqrt{e^{2 x} t^{2}-1}} \int_{0}^{\infty}\left(\mathcal{H}_{0} g^{\dagger}\right)(\xi) \sin \left(\frac{2 e^{2 x} t \xi}{e^{2 x} t^{2}-1}\right) J_{0}\left(\frac{2 e^{x} \xi}{e^{2 x} t^{2}-1}\right) d \xi .
\end{aligned}
$$

Equivalently, the solution may be written as

$$
u(x, t)=\left(\Theta, f^{b}\right)(x, t)-\left(\Upsilon, g^{\dagger}\right)(x, t)
$$

We could write the solution in terms of $1-e^{2 x} t^{2}$ instead of $e^{2 x} t^{2}-1$ in order to avoid the appearance of complex numbers in the solution, but this seems unnatural in this case.

Equations of the form $u_{t t}=(A x+B)^{C} u_{x x}$ may be solved by the same methods. We will not present the full details, but briefly consider the case

$$
u_{t t}=x^{2-2 \alpha} u_{x x}
$$

where $\alpha>1, \alpha \neq 0, \pm 1$. A rescaling of $t$ and an affine transform in $x$ will lead to the more general case. These equations have an infinitesimal symmetry

$$
\mathbf{v}=x t \partial_{x}+\frac{1}{2}\left(\alpha t^{2}+x^{2 \alpha} / \alpha\right) \partial_{t}+\frac{1}{2}(1-\alpha) t \partial_{u} .
$$

Exponentiating and applying the resulting symmetry to $u=1$, we obtain the solution

$$
C(x, t ; \epsilon)=\left(4+4 \alpha \epsilon t+\epsilon^{2}\left(t^{2}-x^{2 \alpha}\right)\right)^{-\beta}, \beta=(\alpha-1) /(2 \alpha) .
$$

The solution

$$
u(x, t)=\int_{0}^{\infty} \varphi(\epsilon) C(x, t ; \epsilon) d \epsilon
$$

can be rewritten using the Laplace transform to produce the operator

$$
\begin{gathered}
u(x, t)=\frac{\sqrt{\pi}\left(\sqrt{t^{2}\left(\alpha^{2}-1\right)+x^{2 \alpha}}\right)^{\frac{1}{2}-\beta}}{2^{2 \beta-1} \Gamma(\beta)\left(t^{2}-x^{2 \alpha}\right)^{\frac{1}{2}}} \int_{0}^{\infty} \Phi(\xi) \exp \left(-\frac{2 \alpha t \xi}{t^{2}-x^{2 \alpha}}\right) \\
\times \xi^{\beta-1 / 2} I_{\beta-\frac{1}{2}}\left(\frac{2 \sqrt{x^{2 \alpha}+t^{2}\left(\alpha^{2}-1\right)} \xi}{t^{2}-x^{2 \alpha}}\right) d \xi .
\end{gathered}
$$

As before, we can absorb the extraneous terms into $\Phi$ and construct two operators which allow us to solve the Cauchy problem for this equation. Again the details are basically as before, involving complexifying the parameter $\xi$ and taking the Hankel transform of the initial data. We leave this to the interested reader.

The methods introduced here can be applied to other classes of problems, such as those with variable wave speed in Ref. 3 or equations of the form

$$
u_{t t}=u_{x x}+f(x) u_{x},
$$


where $f^{\prime}+\frac{1}{2} f^{2}=(a x+b)^{-2}$. For example, the axially symmetric wave equation $u_{t t}=u_{x x}+1 / x u_{x}$ has a symmetry acting on solutions by

$$
\begin{aligned}
\tilde{u}(x, t ; \epsilon) & =\frac{1}{\sqrt{1+2 \epsilon t+\epsilon^{2}\left(t^{2}-x^{2}\right)}} \\
& \times u\left(\frac{x}{1+2 \epsilon t+\epsilon^{2}\left(t^{2}-x^{2}\right)}, \frac{t+\epsilon\left(t^{2}-x^{2}\right)}{1+2 \epsilon t+\epsilon^{2}\left(t^{2}-x^{2}\right)}\right) .
\end{aligned}
$$

Taking the constant solution leads to the pair of solutions for $0<x<t$,

$$
\begin{aligned}
& u(x, t)=\frac{1}{\sqrt{t^{2}-x^{2}}} \int_{0}^{\infty} \xi \Phi(\xi) \cos \left(\frac{t \xi}{t^{2}-x^{2}}\right) J_{0}\left(\frac{x \xi}{t^{2}-x^{2}}\right) d \xi, \\
& v(x, t)=\frac{1}{\sqrt{t^{2}-x^{2}}} \int_{0}^{\infty} \xi \Psi(\xi) \sin \left(\frac{t \xi}{t^{2}-x^{2}}\right) J_{0}\left(\frac{x \xi}{t^{2}-x^{2}}\right) d \xi .
\end{aligned}
$$

Using these we can solve the Cauchy problem. The interested reader can provide the details.

\section{THE WAVE EQUATION IN HIGHER DIMENSIONS}

Let us use return to the task of applying scaling symmetries to the solution of Cauchy problems. We will use a scaling symmetry to solve the Cauchy problem

$$
\begin{aligned}
u_{t t} & =\Delta u-\frac{A}{r^{2}} u, r \neq 0, A>0, \\
u(r, \theta, 0) & =f(r, \theta), \\
u_{t}(r, \theta, 0) & =g(r, \theta) .
\end{aligned}
$$

We will assume that $f, g$ are compactly supported smooth functions. As $A \rightarrow 0$, we recover the solution of the wave equation. This possesses scaling symmetries $u(r, \theta, t) \rightarrow u(\lambda r, \theta, \lambda t)$. Our treatment is similar to that given for the case of parabolic equations in higher dimensions in Ref. 10.

For our solutions, we use separation of variables. Let $u(r, \theta, t)=U(r, \theta) T(t)$. This leads to

$$
U_{r r}+\frac{1}{r} U_{r}+\frac{1}{r^{2}} U_{\theta \theta}-\frac{A}{r^{2}} U=a U, T_{t t}=a T,
$$

where $a$ is the separation constant. We take $a=-1$. So $T(t)=c_{1} \cos t+c_{2} \sin t$. If $U(r, \theta)=R(r)$ $\Theta(\theta)$, then

$$
\Theta\left(R^{\prime \prime}+\frac{1}{r} R^{\prime}\right)+\frac{1}{r^{2}} R \Theta^{\prime \prime}+R \Theta-\frac{A}{r^{2}} R \Theta=0 .
$$

So that

$$
\frac{1}{R}\left(r^{2} R^{\prime \prime}+r R^{\prime}\right)+r^{2}-A=-\frac{1}{\Theta} \Theta^{\prime \prime}=k^{2}
$$

or

$$
r^{2} R^{\prime \prime}+r R^{\prime}+\left(r^{2}-k^{2}-A\right) R=0, \Theta^{\prime \prime}=-k^{2} \Theta .
$$

So $u_{k}(r, \theta, t)=J_{\sqrt{k^{2}+A}}(r) e^{i k \theta} \cos (t)$ and $v_{k}(r, \theta, t)=J_{\sqrt{k^{2}+A}}(r) e^{i k \theta} \sin (t)$ are our solutions. Then, we apply the scaling symmetry. Next, construct solutions of (7.1) by setting

$$
\begin{aligned}
u(r, \theta, t)= & \sum_{k \in \mathbb{Z}} \int_{0}^{\infty} \varphi_{k}(\lambda) J_{\sqrt{k^{2}+A}}(\lambda r) e^{i k \theta} \cos (\lambda t) d \lambda \\
& +\sum_{k \in \mathbb{Z}} \int_{0}^{\infty} \psi_{k}(\lambda) J_{\sqrt{k^{2}+A}}(\lambda r) e^{i k \theta} \sin (\lambda t) d \lambda .
\end{aligned}
$$

Then, we require that

$$
u(r, \theta, 0)=\sum_{k \in \mathbb{Z}} \int_{0}^{\infty} \varphi_{k}(\lambda) J_{\sqrt{k^{2}+A}}(\lambda r) e^{i k \theta} d \lambda=f(r, \theta) .
$$


To this end, we expand $f$ in a Fourier series in $\theta$ so that

$$
f(r, \theta)=\sum_{n \in \mathbb{Z}} \widehat{f}(r, k) e^{i k \theta},
$$

where

$$
\widehat{f}(r, k)=\frac{1}{2 \pi} \int_{0}^{2 \pi} f(r, \phi) e^{-i k \phi} d \phi
$$

This tells us that

$$
\int_{0}^{\infty} \lambda \frac{1}{\lambda} \varphi_{k}(\lambda) J_{\sqrt{k^{2}+A}}(\lambda r) d \lambda=\widehat{f}(r, k)
$$

Inverting the Hankel transform gives

$$
\frac{1}{\lambda} \varphi_{k}(\lambda)=\int_{0}^{\infty} \rho \widehat{f}(\rho, k) J \sqrt{k^{2}+A}(\lambda \rho) d \rho
$$

or

$$
\varphi_{k}(\lambda)=\frac{1}{2 \pi} \int_{0}^{\infty} \int_{0}^{2 \pi} \lambda \rho f(\rho, \phi) e^{-i k \phi} J_{\sqrt{k^{2}+A}}(\lambda \rho) d \phi d \rho .
$$

Similar considerations lead to an expression for $\psi_{k}$, namely,

$$
\psi_{k}(\lambda)=\frac{1}{2 \pi} \int_{0}^{\infty} \int_{0}^{2 \pi} \rho g(\rho, \phi) e^{-i k \phi} J_{\sqrt{k^{2}+A}}(\lambda \rho) d \phi d \rho .
$$

We now consider the $N$ th partial sum of the second series appearing in (7.6). This is

$$
\begin{aligned}
& \sum_{k=-N}^{N} \int_{0}^{\infty} \int_{0}^{\infty} \int_{0}^{2 \pi} \rho g(\rho, \phi) J_{v(k)}(\lambda \rho) J_{v(k)}(\lambda r) e^{i k(\theta-\phi)} \sin (\lambda t) d \phi d \rho d \lambda \\
= & \int_{0}^{\infty} \int_{0}^{\infty} \int_{0}^{2 \pi} \rho g(\rho, \phi) \sin (\lambda t) \sum_{k=-N}^{N} J_{v(k)}(\lambda \rho) J_{v(k)}(\lambda r) e^{i k(\theta-\phi)} d \lambda d \phi d \rho,
\end{aligned}
$$

where we have set $v(k)=\sqrt{k^{2}+A}$. Now since $\left|J_{v(k)}(z)\right| \leq 1$, we have

$$
S=\sum_{k=-\infty}^{\infty} J_{v(k)}(\lambda \rho) J_{v(k)}(\lambda r) e^{i k(\theta-\phi)} \leq \sum_{k=-\infty}^{\infty} J_{v(k)}(\lambda \rho) e^{i k(\theta-\phi)}
$$

and $\sum_{k=-\infty}^{\infty} J_{v(k)}(\lambda \rho) e^{i k(\theta-\phi)}$ and its derivatives are uniformly convergent. See Ref. 10, p. 69 and following. The proof there is more general than our case, but the argument is the same. So the series $S$ and its derivatives converge uniformly.

Now let $z=\frac{r^{2}+\rho^{2}-t^{2}}{2 \rho r}$. By 8.822 of Ref. 16,

$$
P_{\nu(k)-1 / 2}(z)=\frac{1}{\pi} \int_{0}^{\pi} \frac{d \xi}{\left(z+\sqrt{z^{2}-1} \cos \xi\right)^{\nu(k)+1 / 2}} .
$$

Observe that for $\left|z+\sqrt{z^{2}-1} \cos \xi\right|>1$, the series

$$
\sum_{k=-\infty}^{\infty} \frac{e^{i k(\theta-\phi)} d \xi}{\left(z+\sqrt{z^{2}-1} \cos \xi\right)^{\nu(k)+1 / 2}}
$$


is convergent by the comparison test and the limit is bounded in $\xi$. Thus, by the bounded convergence theorem, for $\left|z+\sqrt{z^{2}-1} \cos \xi\right|>1$,

$$
\begin{aligned}
\sum_{k=-\infty}^{\infty} e^{i k(\theta-\phi)} P_{v(k)-1 / 2}(z) & =\lim _{N \rightarrow \infty} \frac{1}{\pi} \sum_{k=-N}^{N} \int_{0}^{\pi} \frac{e^{i k(\theta-\phi)} d \xi}{\left(z+\sqrt{z^{2}-1} \cos \xi\right)^{v(k)+1 / 2}} \\
& =\frac{1}{\pi} \int_{0}^{\pi} \lim _{N \rightarrow \infty} \sum_{k=-N}^{N} \frac{e^{i k(\theta-\phi)} d \xi}{\left(z+\sqrt{z^{2}-1} \cos \xi\right)^{v(k)+1 / 2}} \\
& =\frac{1}{\pi} \int_{0}^{\pi} \sum_{k=-\infty}^{\infty} \frac{e^{i k(\theta-\phi)} d \xi}{\left(z+\sqrt{z^{2}-1} \cos \xi\right)^{v(k)+1 / 2}} .
\end{aligned}
$$

A similar argument holds for $\left|z+\sqrt{z^{2}-1} \cos \xi\right|<1$ using the integral representation

$$
P_{v(k)-1 / 2}(z)=\frac{1}{\pi} \int_{0}^{\pi}\left(z+\sqrt{z^{2}-1} \cos \xi\right)^{v(k)-1 / 2} d \xi
$$

which is the second part of 8.822 in Ref. 16.

So it follows that for $r-\rho<t<r+\rho, r>\rho$ or $\rho-r<t<r+\rho, \rho>r$,

$$
\begin{aligned}
K_{t}(r, \rho, \theta, \phi ; k) & =\int_{0}^{\infty} \sum_{k=-\infty}^{\infty} J_{v(k)}(\lambda \rho) J_{v(k)}(\lambda r) e^{i k(\theta-\phi)} \sin (\lambda t) d \lambda \\
& =\sum_{k=-\infty}^{\infty} e^{i k(\theta-\phi)} \int_{0}^{\infty} J_{v(k)}(\lambda \rho) J_{v(k)}(\lambda r) \sin (\lambda t) d \lambda \\
& =\frac{1}{2 \sqrt{\rho r}} \sum_{k=-\infty}^{\infty} e^{i k(\theta-\phi)} P_{v(k)-1 / 2}\left(\frac{r^{2}+\rho^{2}-t^{2}}{2 \rho r}\right)
\end{aligned}
$$

is a smooth function. If $t<r-\rho, r>\rho$ or $t<\rho-r, \rho>r$, then $K_{t}=0$. If $r+\rho<t$ then a similar argument based on the representation

$$
Q_{v(k)-1 / 2}(z)=\int_{0}^{\infty} \frac{d \xi}{\left(z+\sqrt{z^{2}-1} \cosh \xi\right)^{v(k)+1 / 2}}
$$

(8.823 of Ref. 16) yields

$$
K_{t}(r, \rho, \theta, \phi ; k)=\frac{\cos (v(k) \pi)}{2 \sqrt{\rho r}} \sum_{k=-\infty}^{\infty} e^{i k(\theta-\phi)} Q_{v(k)-1 / 2}\left(-\frac{r^{2}+\rho^{2}-t^{2}}{2 \rho r}\right) .
$$

We then have a solution of (7.1) given by

$$
\begin{aligned}
u_{2}(r, \theta, t) & =\int_{0}^{\infty} \int_{0}^{2 \pi} \rho g(\rho, \phi) K_{t}(r, \rho, \theta, \phi ; k) d \phi d \rho \\
& =\left(g, K_{t}\right),
\end{aligned}
$$

which satisfies $u(r, \theta, 0)=0, \frac{\partial}{\partial t} u_{2}(r, \theta, 0)=g(r, \theta)$. As $g$ is a compactly supported smooth function, this integral converges.

For the first series in (7.6), we obtain a solution satisfying $u(r, \theta, 0)=f(r, \theta), u_{t}(r, \theta, 0)=0$, where $f$ is compactly supported and smooth, given by

$$
u_{1}(r, \theta, t)=\frac{\partial}{\partial t}\left(f, K_{t}\right)
$$

Combining these, we see that

$$
u(r, \theta, t)=\frac{\partial}{\partial t}\left(f, K_{t}\right)+\left(g, K_{t}\right)
$$

is the solution of Cauchy problem (7.1).

In three dimensions, the analysis remains the same, except that we have a double sum involving spherical harmonics in place of the $e^{i k \theta}$ term. As we let $A \rightarrow 0$, this must return the Kirchoff solution of the wave equation, but we do not have a proof of this as yet, so we will not proceed further. 
It should be clear that using scaling symmetries in this way will allow us to construct solutions for a wide variety of linear PDEs.

\section{ELLIPTIC EQUATIONS}

For elliptic equations, we can apply the same methods. Often, however, we may recover a fundamental solution from the corresponding heat kernel. The argument is very well known (for example Ref. 2). If $p(t, x, y)$ is the heat kernel for the elliptic operator $\mathcal{L}$ and the integral converges, then

$$
G(x, y)=-\int_{0}^{\infty} p(t, x, y) d t
$$

satisfies

$$
\begin{aligned}
\mathcal{L} G(x, y) & =-\int_{0}^{\infty} \mathcal{L} p(t, x, y) d t \\
& =-\int_{0}^{\infty} \frac{\partial}{\partial t} p(t, x, y) d t \\
& =p(0, x, y)=\delta(x, y),
\end{aligned}
$$

where $\delta$ is the Dirac delta function. Thus, $G$ is a fundamental solution for $\mathcal{L}$. solution

Example 8.1. The equation $\Delta u+\frac{2 a}{a x+b y} u_{x}+\frac{2 b}{a x+b y} u_{y}=0, x>0, y>0$ has a fundamental

$$
\begin{aligned}
G(x, y, \xi, \eta) & =-\frac{a \xi \log \left(\frac{\left((x+\xi)^{2}+(y-\eta)^{2}\right)\left((x+\xi)^{2}+(y+\eta)^{2}\right)}{\left((x-\xi)^{2}+(y-\eta)^{2}\right)\left((x-\xi)^{2}+(y+\eta)^{2}\right)}\right)}{4 \pi(a x+b y)} \\
- & \frac{b \eta \log \left(\frac{\left((x-\xi)^{2}+(y+\eta)^{2}\right)\left((x+\xi)^{2}+(y+\eta)^{2}\right)}{\left((x-\xi)^{2}+(y-\eta)^{2}\right)\left((x+\xi)^{2}+(y-\eta)^{2}\right)}\right)}{4 \pi(a x+b y)} .
\end{aligned}
$$

To see this, we observe that the PDE

$$
u_{t}=\Delta u+\frac{2 a}{a x+b y} u_{x}+\frac{2 b}{a x+b y} u_{y}=0, x>0, y>0
$$

has a fundamental solution (see Ref. 10) given by

$$
\begin{aligned}
p(t, x, y, \xi, \eta) & =\frac{1}{\pi t(a x+b y)} \exp \left(-\frac{x^{2}+y^{2}+\xi^{2}+\eta^{2}}{4 t}\right) \times \\
& {\left[a \xi \sinh \left(\frac{x \xi}{2 t}\right) \cosh \left(\frac{y \eta}{2 t}\right)+b \eta \cosh \left(\frac{x \xi}{2 t}\right) \sinh \left(\frac{y \eta}{2 t}\right)\right] . }
\end{aligned}
$$

We then have

$$
G(x, y, \xi, \eta)=-\int_{0}^{\infty} p(t, x, y, \xi, \eta) d t
$$

The integral can be evaluated from tables or Mathematica.

Theorem 8.1. Suppose that $K$ is continuous and that the Sturm-Liouville problem,

$$
\begin{aligned}
L^{\prime \prime}(\theta)+(K(\theta)+\lambda) L(\theta) & =0, \\
\alpha_{1} L(a)+\alpha_{2} L^{\prime}(a) & =0, \\
\beta_{1} L(b)+\beta_{2} L^{\prime}(b) & =0
\end{aligned}
$$

has a complete set of eigenfunctions and eigenvalues, and that the eigenvalues are all positive. Then, the elliptic equation

$$
\Delta u+\frac{K(\theta)}{r^{2}} u=0, r>0, \theta \in[a, b]
$$


has a fundamental solution

$$
G(r, \theta, \rho, \phi)=-\sum_{n} L_{n}(\theta) \overline{L_{n}(\phi)} \frac{\rho \cos \left(\sqrt{\lambda_{n}} \sec ^{-1}\left(\frac{2 r \rho}{r^{2}+\rho^{2}}\right)\right)}{2 \sqrt{\lambda_{n}}} .
$$

Proof. In Ref. 10, we studied the initial and boundary value problem

$$
\begin{gathered}
u_{t}=u_{r r}+\frac{1}{r} u_{r}+\frac{1}{r^{2}} u_{\theta \theta}+\frac{K(\theta)}{r^{2}} u, \\
r>0, a \leq \theta \leq b, a, b \in[0,2 \pi], \\
u(r, \theta, 0)=f(r, \theta), f \in \mathcal{D}(\Omega), \\
\alpha_{1} u(r, a, t)+\alpha_{2} u_{\theta}(r, a, t)=0, \\
\beta_{1} u(r, b, t)+\beta_{2} u_{\theta}(r, b, t)=0 .
\end{gathered}
$$

Here, $\Omega=[0, \infty) \times[a, b]$ in polar coordinates. It was established that there is a solution

$$
u(r, \theta, t)=\int_{0}^{\infty} \int_{a}^{b} f(\rho, \phi) p(t, r, \theta, \rho, \phi) \rho d \phi d \rho
$$

where

$$
p(t, r, \theta, \rho, \phi)=\frac{1}{2 t} e^{-\frac{r^{2}+\rho^{2}}{4 t}} \sum_{n} \overline{L_{n}(\phi)} L_{n}(\theta) I_{\sqrt{\lambda_{n}}}\left(\frac{r \rho}{2 t}\right),
$$

in which $L_{n}(\theta), \lambda_{n}, n=1,2,3 \ldots$ are the normalised eigenfunctions and corresponding eigenvalues for the given Sturm-Liouville problem. The series converges uniformly, allowing reversal of the order of integration and summation. Hence, there is a fundamental solution of the given elliptic problem defined by

$$
G(r, \theta, \rho, \phi)=-\int_{0}^{\infty} p(t, r, \theta, \rho, \phi) d t .
$$

Evaluating the integral gives the result.

We can, of course, also obtain solutions of elliptic problems by integrating a test function against a solution obtained by symmetry. We briefly illustrate with the two dimensional Laplace equation which has a rich symmetry group. It is easily verified that if $u$ is a solution of the Laplace equation, then

$$
\tilde{u}(x, y ; \epsilon)=u\left(\frac{x+\epsilon\left(x^{2}+y^{2}\right)}{1+2 \epsilon x+\epsilon^{2}\left(x^{2}+y^{2}\right)}, \frac{y}{1+2 \epsilon x+\epsilon^{2}\left(x^{2}+y^{2}\right)}\right)
$$

is also a solution for $x>0$ and $\epsilon>0$.

As before, one can use this symmetry to construct an integral kernel operator mapping elements of an appropriate function space to harmonic functions. Some properties of these operators are immediate.

Proposition 8.2. Suppose that $u$ and $v$ are harmonic conjugates, so that $u_{x}=v_{y}$ and $u_{y}=-v_{x}$. Then,

$$
\begin{aligned}
& \tilde{u}(x, y ; \epsilon)=u\left(\frac{x+\epsilon\left(x^{2}+y^{2}\right)}{1+2 \epsilon x+\epsilon^{2}\left(x^{2}+y^{2}\right)}, \frac{y}{1+2 \epsilon x+\epsilon^{2}\left(x^{2}+y^{2}\right)}\right), \\
& \tilde{v}(x, y ; \epsilon)=v\left(\frac{x+\epsilon\left(x^{2}+y^{2}\right)}{1+2 \epsilon x+\epsilon^{2}\left(x^{2}+y^{2}\right)}, \frac{y}{1+2 \epsilon x+\epsilon^{2}\left(x^{2}+y^{2}\right)}\right)
\end{aligned}
$$

are also harmonic conjugates.

Proof. This is a straight calculation. For example, 


$$
\begin{aligned}
& \frac{\partial}{\partial x} \tilde{u}(x, y ; \epsilon)=\left(1+2 \epsilon x+\epsilon^{2}\left(x^{2}+y^{2}\right)\right)^{-2} \times \\
& \left(-2 y \epsilon(x \epsilon+1) u_{y}\left(\frac{x+\epsilon\left(x^{2}+y^{2}\right)}{1+2 \epsilon x+\epsilon^{2}\left(x^{2}+y^{2}\right)}, \frac{y}{1+2 \epsilon x+\epsilon^{2}\left(x^{2}+y^{2}\right)}\right)\right. \\
& \left.+T(x, y ; \epsilon) u_{x}\left(\frac{x+\epsilon\left(x^{2}+y^{2}\right)}{1+2 \epsilon x+\epsilon^{2}\left(x^{2}+y^{2}\right)}, \frac{y}{1+2 \epsilon x+\epsilon^{2}\left(x^{2}+y^{2}\right)}\right)\right)
\end{aligned}
$$

and

$$
\begin{aligned}
& \frac{\partial}{\partial y} \tilde{v}(x, y ; \epsilon)=\left(1+2 \epsilon x+\epsilon^{2}\left(x^{2}+y^{2}\right)\right)^{-2} \times \\
& \left(T(x, y ; \epsilon) v_{y}\left(\frac{x+\epsilon\left(x^{2}+y^{2}\right)}{1+2 \epsilon x+\epsilon^{2}\left(x^{2}+y^{2}\right)}, \frac{y}{1+2 \epsilon x+\epsilon^{2}\left(x^{2}+y^{2}\right)}\right)\right. \\
& \left.+2 y \epsilon(x \epsilon+1) v_{x}\left(\frac{x+\epsilon\left(x^{2}+y^{2}\right)}{1+2 \epsilon x+\epsilon^{2}\left(x^{2}+y^{2}\right)}, \frac{y}{1+2 \epsilon x+\epsilon^{2}\left(x^{2}+y^{2}\right)}\right)\right),
\end{aligned}
$$

where we set $T(x, y ; \epsilon)=1+2 \epsilon x+\epsilon^{2}\left(x^{2}-y^{2}\right)$. Since $u_{x}=v_{y}$ and $u_{y}=-v_{x}$, then $\frac{\partial}{\partial x} \tilde{u}(x, y ; \epsilon)=$ $\frac{\partial}{\partial y} \tilde{v}(x, y ; \epsilon)$. The other case is essentially the same.

This has an immediate consequence.

Corollary 8.3. If $u$ and $v$ are harmonic conjugate solutions of the two-dimensional Laplace equation, then so are

$$
U(x, y)=\int_{0}^{\infty} \varphi(\epsilon) u\left(\frac{x+\epsilon\left(x^{2}+y^{2}\right)}{1+2 \epsilon x+\epsilon^{2}\left(x^{2}+y^{2}\right)}, \frac{y}{1+2 \epsilon x+\epsilon^{2}\left(x^{2}+y^{2}\right)}\right) d \epsilon
$$

and

$$
V(x, y)=\int_{0}^{\infty} \varphi(\epsilon) v\left(\frac{x+\epsilon\left(x^{2}+y^{2}\right)}{1+2 \epsilon x+\epsilon^{2}\left(x^{2}+y^{2}\right)}, \frac{y}{1+2 \epsilon x+\epsilon^{2}\left(x^{2}+y^{2}\right)}\right) d \epsilon,
$$

assuming that the integrals are convergent. Consequently, the function $F(z)=U(x, y)+i V(x, y), z$ $=x+i y$ is analytic.

The choice of $\varphi$ for which the integrals are convergent will depend on the choice of $u$ and $v$. For example, take the solution $u(x, y)=y$, apply the symmetry, and integrate against a test function $\varphi$ to obtain

$$
u(x, y)=\int_{0}^{\infty} \frac{y \varphi(\epsilon)}{1+2 \epsilon x+\epsilon^{2}\left(x^{2}+y^{2}\right)} d \epsilon .
$$

Rewriting with the Laplace transform as we did in the previous examples produces the solution

$$
u(x, y)=\int_{0}^{\infty} \Phi(\xi) \exp \left(-\frac{x \xi}{x^{2}+y^{2}}\right) \sin \left(\frac{y \xi}{x^{2}+y^{2}}\right) d \xi
$$

for $x>0$ and $\Phi \in L^{1}([0, \infty))$. By similar means, we can obtain the solution

$$
v(x, y)=\int_{0}^{\infty} \Psi(\xi) \exp \left(-\frac{x \xi}{x^{2}+y^{2}}\right) \cos \left(\frac{y \xi}{x^{2}+y^{2}}\right) d \xi,
$$

and if $\Phi=\Psi$, these solutions are easily shown to be harmonic conjugates

If we take $\Phi(\xi)=\Psi(\xi)=\frac{1}{\Gamma(n+1)} \xi^{n}$, the integrals still converge and we obtain

$$
\begin{aligned}
& u(x, y)=\left(x^{2}+y^{2}\right)^{\frac{n+1}{2}} \sin \left[(n+1) \tan ^{-1}(y / x)\right], \\
& v(x, y)=\left(x^{2}+y^{2}\right)^{\frac{n+1}{2}} \cos \left[(n+1) \tan ^{-1}(y / x)\right] .
\end{aligned}
$$

For $n$ an integer, it is not hard to see that these generate the harmonic polynomials.

To solve the Laplace equation with $u(0, y)=g(y), y>0$, we would let $\Phi(y)=\left(\mathcal{F}_{s}^{-1} g^{\sharp}\right)(y)$, where $g^{\sharp}(y)=g(1 / y)$ and $\mathcal{F}_{s}$ is the Fourier sine transform. We can then proceed to obtain an appropriate function space for $g$. However, this type of problem is unnatural for the Laplace equation. 
Indeed, Cauchy problems are generally ill posed for elliptic equations (see the discussion in Ref. 18) and usually do not have unique solutions. Natural problems for the Laplace equation and elliptic equations in general are boundary value problems, such as those of Dirichlet and Neumann.

Throughout this paper, we have relied upon constructing solutions from trivial solutions. There are many other solutions which we can apply symmetries to, so we can produce a considerable range of integral operators. We can also use other integral transforms. This approach can be used to deduce information about harmonic functions. We conclude with a simple example.

Instead of using the Laplace transform to rewrite our operators, we use the Fourier transform. An easy result may be obtained. We note that $\mathcal{S}(\mathbb{R})$ is the usual Schwartz space.

Proposition 8.4. If $\varphi \in \mathcal{S}(\mathbb{R})$, then

$$
u(x, y)=\frac{1}{2 \pi} \int_{-\infty}^{\infty} \widehat{\varphi}(\xi) \exp \left(-\frac{i x \xi+y|\xi|}{x^{2}+y^{2}}\right) d \xi
$$

is a harmonic function. We take here $\widehat{\varphi}(\xi)=\int_{-\infty}^{\infty} \varphi(r) e^{-i r \xi} d r$.

Proof. We consider the harmonic function

$$
\begin{aligned}
K(x, y ; \epsilon) & =\frac{y}{\pi\left(1+2 \epsilon x+\epsilon^{2}\left(x^{2}+y^{2}\right)\right)} \\
& =\frac{1}{2 \pi} \int_{-\infty}^{\infty} \exp \left(-\frac{i x \xi+y|\xi|}{x^{2}+y^{2}}-i \epsilon \xi\right) d \xi .
\end{aligned}
$$

As $\varphi \in \mathcal{S}(\mathbb{R})$, we also have $\widehat{\varphi} \in \mathcal{S}(\mathbb{R})$ and

$$
\begin{aligned}
u(x, y) & =\int_{-\infty}^{\infty} \varphi(\epsilon) K(x, y ; \epsilon) d \epsilon \\
& =\frac{1}{2 \pi} \int_{-\infty}^{\infty} \int_{-\infty}^{\infty} \varphi(\epsilon) \exp \left(-\frac{i x \xi+y|\xi|}{x^{2}+y^{2}}-i \epsilon \xi\right) d \xi d \epsilon \\
& =\frac{1}{2 \pi} \int_{-\infty}^{\infty} \widehat{\varphi}(\xi) \exp \left(-\frac{i x \xi+y|\xi|}{x^{2}+y^{2}}\right) d \xi .
\end{aligned}
$$

Now we note that $\left(\frac{\partial^{2}}{\partial x^{2}}+\frac{\partial^{2}}{\partial y^{2}}\right) \exp \left(-\frac{i x \xi+y|\xi|}{x^{2}+y^{2}}\right)=0$ and we may differentiate under the integral sign since $\widehat{\varphi}$ has rapid decay.

One may carry out this type of analysis for any linear equation with non-trivial symmetries. We believe that exploring the properties of the group Fourier transform on the symmetry group of a linear PDE may prove to be very fruitful.

${ }^{1}$ M. Abramowitz and I. Stegun, Handbook of Mathematical Functions, with Formulas, Graphs and Mathematical Tables, 10th ed. (Dover, New York, 1972).

${ }^{2}$ R. Beals, B. Gaveau, and E. Greiner, "Complex Hamiltonian mechanics and parametrices for subelliptic Laplacians," Bull. Sci. Math. 121, 1-36 (1997).

${ }^{3}$ G. Bluman and S. Kumei, "On invariance properties of the wave equation," J. Math. Phys. 26(2), 307-318 (1987).

${ }^{4}$ G. Bluman and S. Kumei, Symmetries and Differential Equations (Springer Verlag, 1989).

${ }^{5}$ M. Craddock, "Symmetry groups of partial differential equations, separation of variables and direct integral theory," J. Funct. Anal. 125(2), 452-479 (1994).

${ }^{6}$ M. Craddock, "The symmetry groups of linear partial differential equations and representation theory I," J. Differ. Equations 116(1), 202-247 (1995).

${ }^{7}$ M. Craddock, "The symmetry groups of linear partial differential equations and representation theory : The Laplace and axially symmetric wave equations," J. Differ. Equations 166(1), 107-131 (2000).

${ }^{8}$ M. Craddock, "Fundamental solutions, transition densities and the integration of lie symmetries," J. Differ. Equations 246(1), 2538-2560 (2009).

${ }^{9}$ M. Craddock and A. H. Dooley, "On the equivalence of lie symmetries and group representations," J. Differ. Equations 249(1), 621-653 (2010).

${ }^{10}$ M. Craddock and K. Lennox, "Lie symmetry methods for multidimensional parabolic PDEs and diffusions," J. Differ. Equations 252, 56-90 (2012).

${ }^{11}$ M. Craddock and K. A. Lennox, “Lie group symmetries as integral transforms of fundamental solutions,” J. Differ. Equations 232, 652-674 (2007).

${ }^{12}$ M. Craddock and E. Platen, "Symmetry group methods for fundamental solutions," J. Differ. Equations 207, 285-302 (2004). 
${ }^{13}$ L. Debnath and D. Bhatta, Integral Transforms and Their Applications, 2nd ed. (Chapman and Hall, 2007).

${ }^{14}$ A. Erdelyi, Tables of Integral Transforms, Bateman Manuscript Project Vol. 1 (McGraw Hill, 1954).

15 J. Franco, "Global SL(2,R) representations of the Schrödinger equations with singular potential," Cent. Eur. Math. J. 10(3), 927-941 (2012).

${ }^{16}$ I. S. Gradshteyn and I. M. Ryzhik, Table of Integrals, Series and Products, 6th ed. (Academic Press, 2000).

${ }^{17}$ R. Howe, "On the role of the Heisenberg group in harmonic analysis," Bull. Am. Math. Soc. 3(2), 821-843 (1980).

${ }^{18}$ F. John, Partial Differential Equations, Applied Mathematical Sciences 4th ed. (Springer, Berlin, 1982).

${ }^{19}$ F. W. King, Hilbert Transforms. Volume 1, Encyclopedia of Mathematics and Its Applications Vol. 124 (Cambridge University Press, Cambridge, 2009).

${ }^{20}$ F. W. King, Hilbert Transforms. Volume 2, Encyclopedia of Mathematics and Its Applications Vol. 125 (Cambridge University Press, Cambridge, 2009).

${ }^{21}$ S. Lie, "Zur allgemeinen theorie der partiellen differentialgleichungen beliebeger ordnung," Leipz. Ber. 47, 53-128 (1895).

${ }^{22}$ NIST Handbook of Mathematical Functions, edited by F. W. J. Olver, D. W. Lozier, R. F. Boisvert, and C. W. Clark (Cambridge University Press, Cambridge, 2010).

${ }^{23}$ P. J. Olver, Applications of Lie Groups to Differential Equations, Graduate Texts in Mathematics Vol. 107 (Springer-Verlag, New York, 1993).

${ }^{24}$ A. Robert, Introduction to the Representation Theory of Compact and Locally Compact Groups, London Mathematical Society Lecture Note Series Vol. 80 (Cambridge University Press, Cambridge, 1983).

${ }^{25}$ M. Sepanski and R. Stanke, "On global $S L(2, \mathbb{R})$ symmetries of differential operators," J. Funct. Anal. 224(1), 1-21 (2005).

${ }^{26}$ I. N. Sneddon, Fourier Transforms, International Series in Pure and Applied Mathematics (McGraw Hill, New York, 1951).

${ }^{27}$ E. M. Stein and R. Shakarchi, Functional Analysis. Introduction to Further Topics in Analysis, Princeton Lectures in Analysis Vol. IV (Princeton University Press, Princeton, 2011).

${ }^{28}$ M. Suguira, Unitary Representations and Harmonic Analysis: An Introduction (North Holland, 1990).

${ }^{29}$ E. C. Titchmarsh, An Introduction to the Theory of Fourier Integrals (Clarendon Press, Oxford, 1937).

${ }^{30}$ S. Yakubovich and Y. Luchko, The Hypergeometric Approach to Integral Transforms and Convolutions, Mathematics and Its Applications Vol. 287 (Kluwer, Dordrecht, 1994).

${ }^{31}$ A. Zayed, Handbook of Function and Generalized Function Transformations (CRC Press, 1996).

${ }^{32}$ A. H. Zemanian, Generalized Integral Transformations, Pure and Applied Mathematics Vol. XVIII (Interscience Publishers, 1968). 